\title{
Choice under Uncertainty with the Best and Worst in Mind: Neo-additive Capacities*.
}

\author{
Alain Chateauneuf \\ CERMSEM \\ Université Paris I
}

\author{
Jürgen Eichberger \\ Wirtschaftstheorie I \\ Universität Heidelberg
}

\author{
Simon Grant \\ Department of Economics \\ Rice University
}

September, 2002

This Version: January 7, 2003

\begin{abstract}
The concept of a non-extreme-outcome-additive capacity (neo-additive capacity) is introduced. Neo-additive capacities model optimistic and pessimistic attitudes tow ards uncertainty as observed in many experimental studies. Moreover, neo-additive capacities can be applied easily in economic problems, as we demonstrate by examples. This paper provides an axiomatisation of Choquet expected utility with neo-capacities in a framework of purely subjective uncertainty.
\end{abstract}

\section{JEL Classification: D81}

Keywords: optimism, pessimism, Choquet expected utility, portfolio choice.

\footnotetext{
* We would like to thank Michele Cohen, Peter Hartley, Jean- Yves Jaffray, Mark Machina, Klaus Nehring, Matthew Ryan, Jean-Marc Tallon, and Jean-Christophe Vergnaud for extremely stimulating and helpful discussions.
} 
"That the chance of gain is naturally over-valued we may learn from the universal success of lotteries. [...] The vain hope of gaining some of the great prizes is the sole cause of this demand. The soberest people scarce look upon it as a folly to pay a small sum for the chance of gaining ten or twenty thousand pounds.'

ADAM SMITH (1776)

"The Wealth of Nations" (p.210).

"Overconfidence, however generated, appears to be a fundamental factor promoting the high volume of trade we observe in speculative markets. Without such confidence, one would think that there would be little trading in financial markets.

ROBERT SHILlER (2001)

“Irrational Exuberance” (p.144/5).

\section{Introduction}

Optimism and pessimism are important features of a person's attitude towards uncertainty. On an aggregate level, business cycles and stock market fluctuations have been attributed to "irrational" optimism and pessimism. Economic theory, however, finds it difficult to see in such moods a major factor determining economic behavior. With large amounts of money and wealth at stake, as in the investment behavior of traders in financial markets, one hesitates to attribute major influence on decisions to vague notions of belief.

Faced with uncertainty economists like to think of investors as cool analysts, carefully weighing likelihoods of events relevant for their decisions. Yet, many observers of investment behavior in financial markets, from KEYNES (1921) to RoBERT SHILLER (2001), could not escape the conclusion that psychological effects seem to interact with probabilistic information in shaping investors' behavior.

Embracing RAMSEY's (1926) and DE FINETTI's (1937) personalistic view of probability, SAVAGE (1954) provided a set of behavioral postulates showing that it is possible to view decision makers' behavior in the face of uncertainty as guided by a consistent system of probabilistic beliefs. His axioms gave researchers an opportunity to put these postulates to direct tests. Allais (1953) and ElLSBERG (1962) were the most promi- 
nent articles reporting choice behavior of people which contradicts Savage's postulates. In particular, the Sure-Thing-Principle which allows one to decompose a decision problem, omitting "equivalent parts" and focussing choice on the remaining parts, was quickly identified as especially problematic.

There are behavioral regularities which influence individuals' betting behavior. People distinguish categorically between situations which they consider as certain, just possible, or strictly impossible. These consistently observed certainty and impossibility effects cannot be modeled by a transition from zero probability of an event to a positive probability, or from a positive probability to the probability of one.

A typical lottery with a high prize on a very unlikely event can turn the certainty of low wealth for a poor person into the possibility of great riches, providing a reason for accepting an unfair gamble. Conversely, rich people may find the possibility of loosing substantial amounts of wealth so dangerous that high expected returns are necessary to induce them to an investment.

BELL (1985) interprets these psychological biases as disappointment aversion or elationseeking behavior. He studies situations where these biases determine the behavior, such as the process of releasing information, behavior in auctions and the Ellsberg paradox. Based on these observations he argues for an inverse-S shaped pattern of decision weights as an adequate representation of individual attitudes towards uncertainty.

Optimistic behavior overestimates the likelihood of good outcomes while pessimistic attitudes exaggerate the likelihood of bad outcomes. Based upon mounting experimental evidence for certainty and impossibility effects, WAKKER (2001) extends these notions to arbitrary events with rank-ordered outcomes and characterizes optimistic and pessimistic attitudes. In the context of the Choquet expected utility (CEU) model, concave capacities reflect optimistic attitudes towards uncertainty, while convex capacities model pessimism.

\subsection{Experimental evidence}

CAMERER (1995) reviews numerous studies refuting the validity of the expected utility 
approach as a description of individual behavior. More recently, however, experiments find evidence for typical patterns of deviation from the expected utility model. In particular, one often observes subjects willing to bet on high outcomes with low probability while refusing to accept even small risks. For subjects choosing between lotteries, one can explain such behavior by a function $w(p)$ weighting the probability $p$ of events. Experimental studies by Gonzalez \& Wu (1999), Abdellaoui (2000), Bleichrodt \& PinTo (2000) and others show a pattern of probability weights as in Figure 1.

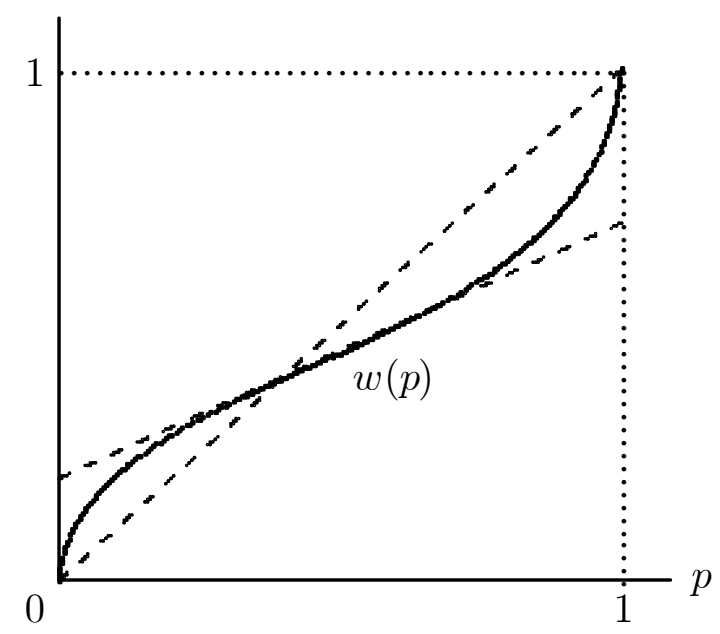

Figure 1: Probability weighting function

The decision weight of an event $E, w(p(E))$, measured by the willingness to bet on this event, differs usually from the probability of the event $p(E)$. Figure 1 shows an inverse-S shaped weighting function, overweighting probabilities close to zero and underweighting probabilities close to one as. TVERSKY \& WAKKER (1995) study the relationship between decision weights and attitudes towards risk and characterize the possibility and certainty effects. WAKKER (2001) defines optimism and pessimism in terms of decision weights. This article contains also a brief survey of the relevant experimental literature. KILKA \& WEBER (2001) demonstrate how decision weights and subjective probabilistic beliefs can be distinguished in experiments. 


\subsection{Axiomatic treatments}

A simple version of an inverse-S shaped weighting function $w(p)$,

$$
w(p):=\left\{\begin{array}{ll}
1 & \text { for } p=1 \\
\lambda+(1-\lambda-\gamma) \cdot p & \text { for } 0<p<1 \\
0 & \text { for } p=0
\end{array},\right.
$$

is depicted in Figure 2.

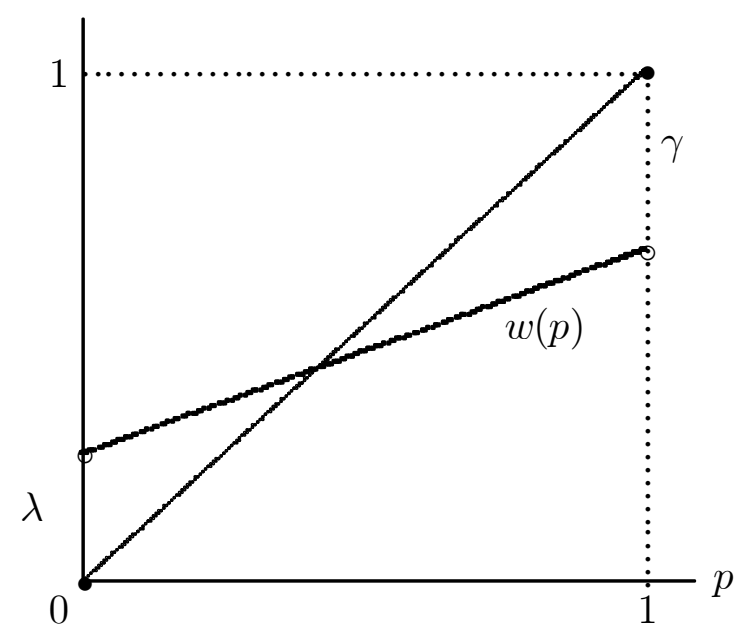

Figure 2: Neo-additive capacity

This weighting scheme, which we call neo-additive capacity for reasons explained below, has a simple Choquet integral. Integrating over a lottery yields a weighted average of the expected utility of the lottery and its maximal and minimal outcomes.

In the context of choice of objective lotteries there are a few studies providing behavioral axioms for functionals which combine expected utility with minimum utility and maximum utility approach. GILBOA (1988) and JAFFRAY (1988) independently axiomatize a functional which aggregates the expected utility functional and the minimum utility functional in a monotonic function. Their representations capture only pessimistic attitudes towards uncertainty. CoHEN (1992), in contrast, provides axioms for a representation of preferences over lotteries as a weighted average of expected utility, minimum utility and maximum utility. For the context of choice over lotteries, her paper provides the behavioral foundations for the representation studied in this paper. To our knowledge 
there is no axiomatization for choice over acts in either the Anscombe-Aumann or the Savage framework.

The neo-additive weighting scheme provides an easy way to model the certainty and the impossibility effects. Combined with a probability function $\operatorname{Pr}($.$) over ranges of$ monetary outcomes, this weighting scheme models an individual who overweights the likelihood of a monetary outcome $x$ exceeding $\bar{x}$,

$$
\begin{aligned}
w(\operatorname{Pr}(x & >\bar{x}))=\lambda+(1-\lambda-\gamma) \cdot \operatorname{Pr}(x>\bar{x}) \\
& >\operatorname{Pr}(x>\bar{x})
\end{aligned}
$$

whenever $\operatorname{Pr}(x>\bar{x})<\lambda /(\lambda+\gamma)$. In contrast, outcomes below $\underline{x}$ occurring with low probability $\operatorname{Pr}(x \leq \underline{x})$ obtain a weight,

$$
\begin{aligned}
\bar{w}(\operatorname{Pr}(x & \leq \underline{x})) \equiv 1-w(1-\operatorname{Pr}(x>\underline{x})) \\
& =(1-\lambda)-(1-\lambda-\gamma)(\operatorname{Pr}(x \leq \underline{x})) \\
& >\operatorname{Pr}(x \leq \underline{x}),
\end{aligned}
$$

whenever $\operatorname{Pr}(x \leq \underline{x})<\gamma /(\lambda+\gamma)$.

In the next section, we introduce some notation and concepts necessary for our analysis. Section 3 studies the neo-additive weighting scheme in the context of the CEU model. This parameterized CEU model can be easily applied to economic models in order to analyse the implications of the certainty and impossibility effect. Section 4 illustrates the potential of the neo-additive $\mathrm{CEU}$ representation for economic applications in the context of a portfolio choice model. Section 5 provides an axiomatic treatment of neo-additive capacities in a framework of purely subjective uncertainty. Proofs are collected in an appendix.

\section{Capacities and the Choquet integral}

We assume that the uncertainty a decision maker faces can be described by a non-empty set of states, denoted by $S$. This set may be finite or infinite. Associated with the set of states is the set of events, taken to be a sigma-algebra of subsets of $S$, denoted by $\mathcal{E}$. 
We assume that for each $s$ in $S,\{s\}$ is in $\mathcal{E}$. Capacities are real-valued functions defined on $\mathcal{E}$, that generalize the notion of probability distributions. Formally, a capacity is a normalized monotone set function.

Definition 2.1 A capacity is a function $\nu: \mathcal{E} \rightarrow \mathbb{R}$ which assigns real numbers to events, such that

$$
\begin{array}{ll}
\text { (i) } \quad E, F \in \mathcal{E}, E \subseteq F \text { implies } \nu(E) \leqslant \nu(F), & \text { monotonicity } \\
\text { (ii) } \quad \nu(\emptyset)=0 \text { and } \nu(S)=1 . & \text { normalization }
\end{array}
$$

A capacity $\nu$ is called convex if $\nu(E \cup F) \geq \nu(E)+\nu(F)-\nu(E \cap F)$ holds for arbitrary events $E, F \in \mathcal{E}$. If the reverse inequality holds then the capacity is called concave. Probability distributions are special cases of capacities which are both concave and convex. For each capacity $\nu$ there is a dual or conjugate capacity $\bar{\nu}$ defined by $\bar{\nu}(E)=1-$ $\nu(S-E)$ for all $E \in \mathcal{E}$. If the dual capacity $\bar{\nu}$ is convex, then the capacity $\nu$ is concave. The most common way to integrate functions with respect to a capacity is the Choquet integral. Let $f: S \rightarrow \mathbb{R}$ be a $\mathcal{E}$-measurable real-valued function. We consider finite outcome acts and suppose that $f$ has finite range, that is, the set $f(S)$ is finite. We call a function $f$ with these properties a simple function. The Choquet integral can therefore be written in the following intuitive form.

Definition 2.2 For any simple function $f$ the Choquet integral with respect to the capacity $\nu$ is defined as

$$
V(f \mid \nu)=\sum_{u \in f(S)} u \cdot[\nu(\{s \mid f(s) \geq u\})-\nu(\{s \mid f(s)>u\})] .
$$

Each element in the range has a decision weight equal to the difference between the capacity of the states yielding an element better or equal than the one under consideration and the capacity of the states yielding a strictly better outcome. The Choquet integral is interpreted as the expected value of the function $f$ with respect to the capacity $\nu$. The decision weights used in the computation of the Choquet integral will overweight high outcomes if the capacity is concave and will overweight low outcomes if the capacity is convex. It is therefore well-suited to model such responses to ambiguity as optimism or pessimism. 
SARIN \& WAKKER (1998) provide a detailed discussion of decision weights.

\section{Neo-additive capacities}

Choquet expected utility (CEU) maximisers will never bet on events $E \in \mathcal{E}$ with capacity value $\nu(E)=0$, nor will they bet against events $F \in \mathcal{E}$ with capacity value $\nu(F)=1$. An event with capacity value zero will therefore never influence the choice of a CEU decision maker and can be called a null event. Similarly, an event with capacity value of one will be called universal event. For probability distributions, the complement of a null event will necessarily be a universal event. For general capacities, this is not true except for the empty set and its complement, the state space $S$. As Example 3.1 below illustrates, it is useful to allow for other null events with complements which are universal.

Let $Z \in \mathcal{E}$ be the largest null event in $\mathcal{E}$ such that $S-Z$ is universal. Since capacities are monotonic, every event $E \subset Z$ must also be a null event and $S-E$ must be universal. All these events will have a capacity value of zero and the capacity value of their complement is one. In many cases, $Z=\emptyset$ is the largest set with these properties.

In this section we introduce a special kind of capacity which we call a neo-additive capacity because it is additive on non-extreme outcomes, if these occur on essential events. Neo-additive capacities can be viewed as a convex combination of an additive capacity and two capacities, one of which reflects complete ignorance or complete ambiguity, denoted $\mu^{0}$, and another which reflects complete confidence, denoted $\mu^{1}$.

Definition 3.1 For all $E \in \mathcal{E}$,

$$
\mu^{0}(E):=\left\{\begin{array}{ll}
1 & \text { for } S-E \subseteq Z \\
0 & \text { otherwise }
\end{array} \quad, \mu^{1}(E):=\left\{\begin{array}{ll}
0 & \text { for } E \subseteq Z \\
1 & \text { otherwise }
\end{array} .\right.\right.
$$

The capacity $\mu^{0}$ is convex. The capacity $\mu^{1}$ is the conjugate of $\mu^{0}$, that is $\mu^{1}(E)=1-$ $\mu^{0}(S-E)$ for all $E$ in $\mathcal{E}$, and therefore is concave.

Let $\pi$ be a finitely additive probability distribution defined over $\mathcal{E}$, such that $\pi(A)=0$ for all $A \subseteq Z$ and $\pi(B)=1$ for all $S-B \subseteq Z$. Denote by $\Delta:=\{(\alpha, \beta) \mid \alpha \geq 0$, $\beta \geq 0, \alpha+\beta \leq 1\}$ the simplex in $\mathbb{R}^{2}$. 
Definition 3.2 For a given finitely additive probability distribution $\pi$ on $(S, \mathcal{E})$ with $\pi(E)=0$ for $E \subseteq Z$, and a pair of numbers $(\gamma, \lambda) \in \Delta$, a neo-additive capacity $\nu(\cdot \mid \pi, \gamma, \lambda)$ is defined as

$$
\nu(E \mid \pi, \gamma, \lambda):=\gamma \cdot \mu^{0}(E)+\lambda \cdot \mu^{1}(E)+(1-\gamma-\lambda) \cdot \pi(E)
$$

for all $E$ in $\mathcal{E}$.

It is straightforward to derive the Choquet integral of a simple function $f$ with respect to a neo-additive capacity in terms a weighted sum of the act's infimum over $S-Z$, its supremum over $S-Z$ and its expectation with respect to $\pi$.

Lemma 3.1 The Choquet expected value of a simple function $f: S \rightarrow \mathbb{R}$ with respect to the neo-additive capacity $\nu(E \mid \pi, \gamma, \lambda)$ is given by:

$$
V(f \mid \nu(\cdot \mid \pi, \gamma, \lambda)):=\gamma \cdot \inf _{S-Z} f+\lambda \cdot \sup _{S-Z} f+(1-\gamma-\lambda) \cdot \mathrm{E}_{\pi}[f] .
$$

Proof. To see this note that $V\left(f \mid \mu^{0}\right)=\inf _{S-Z} f V\left(f \mid \mu^{1}\right)=\sup _{S-Z} f$ and $V(f \mid \pi)=\mathrm{E}_{\pi}[f]$. The result then follows from the linearity of the Choquet integral with respect to the capacity (Denneberg (2000), Properties (ix) and (x) on page 49).

Notice that we do not require that $\pi(E)=0$ implies $E$ to be null. Indeed nothing in the definitions above rule out $Z=\varnothing$, and hence $S$ to be the only universal event in $\mathcal{E}$. In this case, if for some event $E \neq \emptyset, \pi(E)=0, \nu(E)=\lambda$ is positive and $\nu(S-E)=1-\gamma$ is less than one. That is, an event $E$ may receive a zero decision weight in the evaluation of the Choquet expected value for any act with neither its infimum nor its supremum on $E$. If the infimum (respectively, supremum) occurs in a state of $E$, however, the decision weight on $E$ is $\gamma$ (respectively, $\lambda$ ). For further illustration of this point, consider the following example.

Example 3.1 Suppose $S$ is the unit interval, $[0,1], \mathcal{E}$ is the Borel sigma algebra and $\pi$ a uniform distribution. If a decision maker is only willing to bet on states in [0.5, 1], considering states in $[0,0.5)$ impossible, one can model such beliefs and behaviour by the maximal null event $Z=[0,0.5)$ and $\pi$ the uniform distribution on $[0.5,1]$. Clearly, 
$\pi(s)=0$ for all $s \in S$, yet $\nu(s)=0$ for $s \in[0,0.5)$ and $\nu(s)=\lambda$ for $s \in[0.5,1]$. Moreover, though $\pi(S-\{s\})=1$ for all $s \in S$, we have $\nu(S-\{s\})=1-\gamma$ for all $s \in[0.5,1]$, since $S-(S-\{s\})=\{s\} \nsubseteq Z$ in this case.

Example 3.1 shows also that an event as $\{s\}$ may matter for the decision maker if the best or worst outcome is associated with it, even if it has probability zero.

Several well-known decision criteria can be viewed as special cases of the Choquet integral of a neo-additive capacity:

$$
\begin{aligned}
& \text { (i) } \gamma=\lambda=0 \quad \text { expected utility, } \\
& \text { (ii) } 1 \geq \gamma>0, \lambda=0 \text { pure pessimism, } \\
& \text { (iii) } \gamma=0,1 \geq \lambda>0 \text { pure optimism, } \\
& \text { (iv) } \gamma+\lambda=1 \quad \text { Hurwitz criterion. }
\end{aligned}
$$

Neo-additive capacities satisfy three conditions:

- They are additive for pairs of events which are not null and do not form a partition of a universal event.

- They exhibit uncertainty aversion for some events.

- They exhibit uncertainty preference for some other events.

Indeed, as the following proposition shows, these conditions characterize neo-additive capacities completely.

Proposition 3.1 Let $\nu$ be a capacity on $(S, \mathcal{E})$, where $\mathcal{E}$ contains at least three pair wise disjoint events $E_{1}, E_{2}$ and $E_{3}$ with $0<\nu\left(E_{i}\right)<1$ for $i=1,2,3$. Then the following statements are equivalent:

(i) $\nu$ is a neo-additive capacity,

(ii) the capacity $\nu$ satisfies the following properties:

(a) for any three events $(E, F, G) \in \mathcal{E} \times \mathcal{E} \times \mathcal{E}$ such that $E \cap F=\emptyset=E \cap G$, $\nu(E \cup F)<1$ and $\nu(E \cup G)<1$,

$$
\nu(E \cup F)-\nu(F)=\nu(E \cup G)-\nu(G),
$$

(b) for some $(E, F) \in \mathcal{E} \times \mathcal{E}$ such that $E \cap F=\emptyset$ and $\nu(E \cup F)<1$,

$$
\nu(E \cup F) \leq \nu(E)+\nu(F)
$$


(c) for some $(E, F) \in \mathcal{E} \times \mathcal{E}$ such that $E \cap F=\emptyset$ and $\nu(E \cup F)<1$,

$$
\bar{\nu}(E \cup F) \leq \bar{\nu}(E)+\bar{\nu}(F) .
$$

Proof. Appendix.

Note that for a neo-additive capacity $\nu$ on $(S, \mathcal{E})$, where $\mathcal{E}$ contains at least three nonnull events, as ass umed throughout the paper, uniqueness of the pessimism and optimism coefficients $(\gamma, \lambda)$ and of the underlying probability measure $\pi$ is guaranteed. This is proved in a lemma preceding the proof of Proposition 3.1 in the appendix.

Property (iia) establishes additivity of the neo-additive capacity for events that yield nonextreme outcomes. According to property (iib), the capacity overweights the event in which the most preferred prize is obtained, hence $\lambda \geq 0$. Property (iic) says the capacity overweights also the event with the least preferred prize. It implies $\gamma \geq 0$.

\subsection{Optimism and pessimism}

In this section we will provide two arguments why one may be justified to interpret the overweighting of the extreme outcomes with the notions of optimism and pessimism. The first argument shows that neo-additive capacities are a special case of the behavioural concept of optimism and pessimism advanced in WAKKER (2001). The second argument appeals to the intuitive notion of optimism and pessimism suggested by the context of the multiple prior approach.

\subsubsection{The behavioural approach of Wakker (2001)}

Inspired by the Allais and Ellsberg paradox, WAKKER (2001) suggests a notion of optimism and pessimism based on choice behaviour over acts. This approach derives its appeal from its immediate testability in experiments and its natural representation by properties of capacities.

Properties (iib) and (iic) of Proposition 3.1 imply the neo-additive capacity to be concave on some events, which corresponds to the notion of optimism suggested in WAKKER (2001), and convex on some others, hence pessimistic in the sense of WAKKER (2001). To see this, consider the following four acts $f_{1}, f_{2}, f_{3}, f_{4}$ defined on a partition of the state space $(B, A, I, L)$ with real outcomes $M>m>0$. 


\begin{tabular}{l|l|l|l|l|} 
& $B$ & $A$ & $I$ & $L$ \\
\hline$f_{1}$ & $M$ & $m$ & $m$ & 0 \\
\hline$f_{2}$ & $M$ & $M$ & 0 & 0 \\
\hline$f_{3}$ & $m$ & $m$ & $m$ & $m$ \\
\hline$f_{4}$ & $m$ & $M$ & 0 & $m$ \\
\hline
\end{tabular}

Assume that $m$ is chosen such that $V\left(f_{1}\right)=V\left(f_{2}\right)$. WAKKER (2001) calls a decision maker pessimistic if $V\left(f_{3}\right)>V\left(f_{4}\right)$ and optimistic if $V\left(f_{3}\right)<V\left(f_{4}\right)$. Of course, an expected utility maximiser must be indifferent between $f_{3}$ and $f_{4}$.

If the decision maker is indifferent between acts $f_{1}$ and $f_{2}$, then $m$ measures the willingness to pay for the gamble $M$ on $A$ and 0 on $I$ conditional on the gamble $M$ on $B$ and 0 on $L$. If $f_{3}$ is preferred to $f_{4}$, then the gamble $M$ on $A$ and 0 on $I$ is worth less than $m$ because there is no chance of losing in event $L$. This special attention given to bad outcomes is associated with pessimism. In contrast, an optimist, will be willing to pay more for the gamble $M$ on $A$ and 0 on $I$ if there is no chance of winning in event $B$.

Consider a neo-additive capacity $\nu(\cdot \mid \pi, \gamma, \lambda)$. From $V\left(f_{1} \mid \nu(\cdot \mid \pi, \gamma, \lambda)\right)=V\left(f_{2} \mid \nu(\cdot \mid \pi, \gamma, \lambda)\right)$, we conclude that

$$
(1-\gamma-\lambda) \cdot[m \cdot \pi(A)+m \cdot \pi(I)]=(1-\gamma-\lambda) \cdot M \cdot \pi(A) .
$$

It turns out that neo-additive capacities exhibit pessimism for some acts and optimism for others.

Case (i): pessimism

$V\left(f_{3} \mid \nu(\cdot \mid \pi, \gamma, \lambda)\right)>V\left(f_{4} \mid \nu(\cdot \mid \pi, \gamma, \lambda)\right)$ implies $\gamma>0$, since simple computations show that $V\left(f_{3} \mid \nu(\cdot \mid \pi, \gamma, \lambda)\right)>V\left(f_{4} \mid \nu(\cdot \mid \pi, \gamma, \lambda)\right)$ if and only if $m \cdot(\gamma+\lambda)>\lambda \cdot M$. Hence, $\gamma>0$. Moreover, it is straightforward that $V\left(f_{3} \mid \nu(\cdot \mid \pi, \gamma, \lambda)\right)>V\left(f_{4} \mid \nu(\cdot \mid \pi, \gamma, \lambda)\right)$ for any $M>m>0$ is equivalent to $\lambda=0$ and $\gamma>0$, i.e., pure pessimism.

Case (ii): optimism

Similarly, $V\left(f_{3} \mid \nu(\cdot \mid \pi, \gamma, \lambda)\right)<V\left(f_{4} \mid \nu(\cdot \mid \pi, \gamma, \lambda)\right)$ implies $\lambda>0$, and $V\left(f_{3} \mid \nu(\cdot \mid \pi, \gamma, \lambda)\right)<$ $V\left(f_{4} \mid \nu(\cdot \mid \pi, \gamma, \lambda)\right)$ for any $M>m>0$ is equivalent to $\gamma=0$ and $\lambda>0$, i.e., pure optimism. 
Neo-additive capacities show both optimism and pessimism as they relate to the certainty and the impossibility effect ${ }^{1}$.

\subsubsection{The multiple-prior approach}

The multiple-prior approach assumes that uncertainty of a decision maker can be represented by a set of probability distributions $\mathcal{D}$. The evaluation of an act is the expected utility with respect to the probability distributions in the set $\mathcal{D}$. Attitudes towards the ambiguity about the correct probability distribution can then be captured by the weights which the decision maker puts on the best or worst expected utility over the set $\mathcal{D}$.

Without loss of generality, but for the ease of exposition, assume there is a finite set of states $S=\{1, \ldots, n\}$. The set of probability distributions over $S$ is the simplex $\Delta^{n}$ in this case. Let the set of probability distributions $\mathcal{D} \subseteq \Delta^{n}$, which the decision maker considers possible, be compact and convex. In the multiple-prior approach preferences over acts $\left(x_{1}, \ldots, x_{n}\right)$ are represented by the preference functional

$$
\mathcal{V}\left(x_{1}, \ldots, x_{n} \mid \alpha, \mathcal{D}\right):=\alpha \cdot \min _{\widetilde{\pi} \in \mathcal{D}}\left[\sum_{s \in S} u\left(x_{s}\right) \cdot \widetilde{\pi}_{s}\right]+(1-\alpha) \cdot \max _{\widetilde{\pi} \in \mathcal{D}}\left[\sum_{s \in S} u\left(x_{s}\right) \cdot \widetilde{\pi}_{s}\right] .
$$

The parameter $\alpha \in[0,1]$ represents the weight given to the worst expected utility and $(1-\alpha)$ is the weight given to the best expected utility. Optimism corresponds to the weight given the best expected utility and pessimism to the weight given the worst expected utility. A nice feature of this approach is the natural separation between ambiguity, reflected by the set $\mathcal{D}$, and attitudes towards ambiguity, reflected by the degrees of pessimism $\alpha$ and optimism $(1-\alpha)$.

The Choquet expected utility of a neo-additive capacity defined in Equation 1 can be viewed as a multiple-prior expected utility. Applied to the case of a finite state space, it is not difficult to verify the following equality:

$$
V\left(x_{1}, \ldots, x_{n} \mid \nu(\cdot \mid \pi, \gamma, \lambda)\right):=
$$

1 They are cavex in the sense of WAKKER (2001, P. 1049), that is concave for events which are revealed unlikely and convex for events which are revealed as likely. 


$$
\begin{aligned}
& \gamma \cdot \min _{s \in S} u\left(x_{s}\right)+\lambda \cdot \max _{s \in S} u\left(x_{s}\right)+(1-\gamma-\lambda) \cdot\left[\sum_{s \in S} u\left(x_{s}\right) \cdot \pi_{s}\right] \\
= & \frac{\gamma}{\gamma+\lambda} \cdot \min _{\widetilde{\pi} \in \mathcal{D}}\left[\sum_{s \in S} u\left(x_{s}\right) \cdot \widetilde{\pi}_{s}\right]+\frac{\lambda}{\gamma+\lambda} \cdot \max _{\widetilde{\pi} \in \mathcal{D}}\left[\sum_{s \in S} u\left(x_{s}\right) \cdot \widetilde{\pi}_{s}\right] \\
= & : \mathcal{V}\left(x_{1}, \ldots, x_{n} \mid \frac{\gamma}{\gamma+\lambda}, \mathcal{D}\right),
\end{aligned}
$$

with

$$
\mathcal{D}:=\left\{\widetilde{\pi} \in \Delta^{n} \mid \tilde{\pi}_{s} \geq(1-\gamma-\lambda) \cdot \pi_{s}, s \in S\right\} .
$$

Note that the set of probabilities $\mathcal{D}$ is convex and compact. Moreover, it has a nice geometric structure as Figure 3 illustrates for the case of $n=3$. For a neo-additive capacity

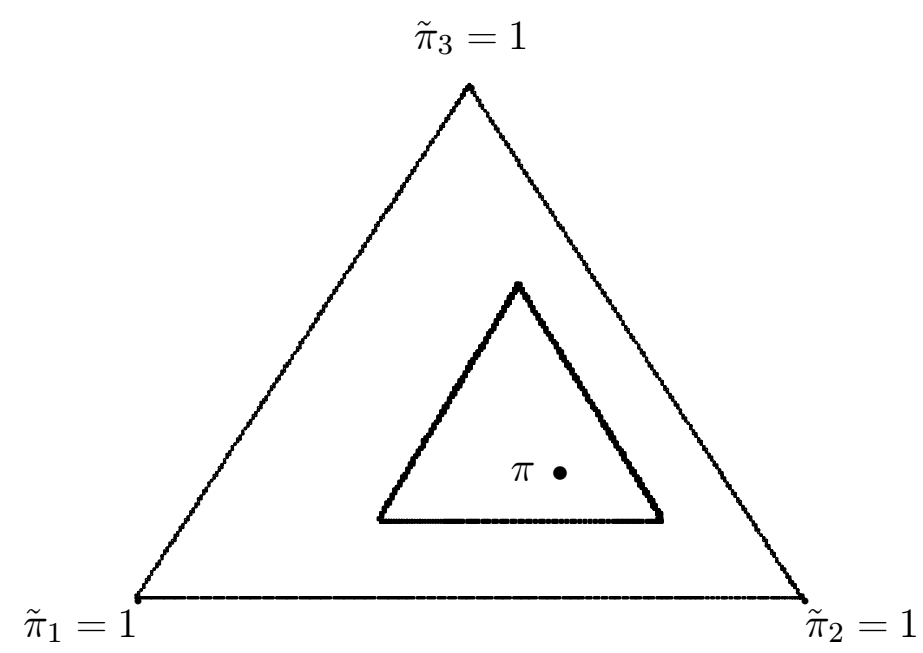

Figure 3: Multiple priors

$\nu(\cdot \mid \pi, \gamma, \lambda)$ the set of possible probability distributions $\mathcal{D}$ is centered around the probability distribution $\pi$ and has a size determined by $\gamma+\lambda$. Hence, $\gamma+\lambda$ can be viewed as the degree of ambiguity about the additive probability distribution $\pi$. The degree of pessimism $\frac{\gamma}{\gamma+\lambda}$ and the degree of optimism $\frac{\lambda}{\gamma+\lambda}$ measure the decision maker's attitude towards this ambiguity. Thus, neo-additive capacities have also a natural interpretation in the context of the multiple-prior approach.

It is well-known that the Choquet expected utility approach is equivalent to the multiple 
prior approach if capacities are convex. In general however, neo-additive capacities are neither convex nor concave. The multiple-prior representation of the Choquet integral of neo-additive capacities is a special case of the more general multiple prior-representation axiomatised recently by GHIRARDATO, MACCHERONI \& MARINACCI (2002) and is similar to the $\alpha$-maxmin expected utility approach of KOPYLOV (2002).

\section{Economic Applications}

The ups and downs of economic activity during the business cycle which are usually accompanied by swings in investors' sentiments, ranging from bull to bear spirits in financial markets, provide numerous examples of the impact of uncertainty on economic behavior.

Neo-additive capacities provide a natural way for modelling optimism and pessimism influencing economic activities. The parameters of a neo-additive capacity can be interpreted as measuring confidence in beliefs and degrees of optimism and pessimism. A neo-additive capacity $\nu(E \mid \pi, \gamma, \lambda)$ is based on an additive probability distribution $\pi$ reflecting the subjective beliefs of the decision maker. It represents an assessment of the likelihood of events consistent with the individual's belief. The weight $(1-\gamma-\lambda)$ given to $\pi$ is a measure of the degree of confidence which the individual holds in this belief. The core belief of a neo-additive capacity represented by the additive probability distribution $\pi$ can be determined endogenously in equilibrium ${ }^{2}$. Thus, standard equilibrium analysis is always the special case of full confidence, $\gamma=\lambda=0$.

Positive parameters $\gamma$ and $\lambda$ represent the impact of pessimism and optimism respectively. Neo-additive capacities can therefore model psychological phenomena such as excessive optimism and pessimism which have been put forward as explanations for economic behavior in depressions or bubbles and which have been confirmed in laboratory experiments.

In this section we show by example that optimism and pessimism can explain behavior

2 EichBerger \& KELSEy (2000) provide a thorough analysis of strategic games when beliefs are modelled as non-additive capacities. 
inconsis tent with expected utility maximization. In theses cases, optimis $m$ and pessimism can help to explain well-known economic puzzles. We will reconsider the paradox of people buying insurance and gambling, and we will review portfolio choice behavior where one observes unreasonably high risk premia (the equity premium puzzle) and a willingness to invest in high-risk stock of unknown start-up companies (the small stock puzzle).

\subsection{Insurance and gambling}

The same individual is often observed to buy both insurance against risk and lottery tickets. As our introductory quotation of Adam Smith illustrates, such behavior is ubiquitous but hard to reconcile with rational decision making based on probabilistic calculus. For expected utility maximizers with a von Neumann-Morgenstern utility function such behavior is hard to explain. ${ }^{3}$ Buying insurance suggests a preference for reduced risk, while paying for a lottery implies preference for a risky gamble, often at very unfair odds.

To see how both types of behavior can be accommodated by a neo-additive capacity, consider an individual endowed with wealth $x$, whose preferences over lotteries can be represented by the Choquet expected utility of a neo-additive capacity, with parameters $\gamma>\lambda \geq 0$ for the neo-additive capacity and utility index $u$ (taken to be concave). This individual faces a (small) probability $\pi_{L}$ of incurring a loss of size $L$. Insurance coverage is available at a premium $q$. Also available at a price $p$ is a lottery ticket that 'wins' with (a very small ) probability $\pi_{W}$ and pays out the single prize of size $W$ and otherwise pays out nothing. Suppose that the individual views the event in which he incurs the loss and the event in which he wins the lottery (should he purchase a ticket) are independent. Further suppose that $\left[\gamma+(1-\gamma-\lambda) \cdot \pi_{L}\right] \cdot L>q \geq \pi_{L} \cdot L$. The weak inequality is a feasibility condition for the insurance premium to cover at least the expected loss (and if strict it means that the insurance coverage is actuarially unfair). The strict inequality is satisfied if the individual has a positive degree of pessimism $\gamma$ and if the potential loss

3 Friedman \& SAVAGE (1948) suggest an S-shaped von Neumann-Morgenstern utility function. This approach to reconcile such behaviour has been critisised by MARKOWITZ (1952). See HiRSHLEIFER \& RILEY (1992) for a discussion of the Friedman-Savage approach (pp. 26-28). 
$L$ is sufficiently large.

The difference in the Choquet expected utilities between buying and not buying the insurance is

$$
\begin{aligned}
& u(x-q)-\left(\left[\lambda+(1-\gamma-\lambda) \cdot\left(1-\pi_{L}\right)\right] \cdot u(x)+\left[\gamma+(1-\gamma-\lambda) \cdot \pi_{L}\right] \cdot u(x-L)\right) \\
\geq & u(x-q)-u\left(\left[\lambda+(1-\gamma-\lambda) \cdot\left(1-\pi_{L}\right)\right] \cdot x+\left[\gamma+(1-\gamma-\lambda) \cdot \pi_{L}\right] \cdot(x-L)\right) \\
= & u(x-q)-u\left(x-\left[\gamma+(1-\gamma-\lambda) \pi_{L}\right] L\right)>0
\end{aligned}
$$

The first inequality follows from Jensen's inequality applied to the convex function $-u$, and the second inequality follows from monotonicity of $u$. Figure 4 illustrates the desirability of purchase of full coverage at the unfair premium for the case where $u$ is affine.

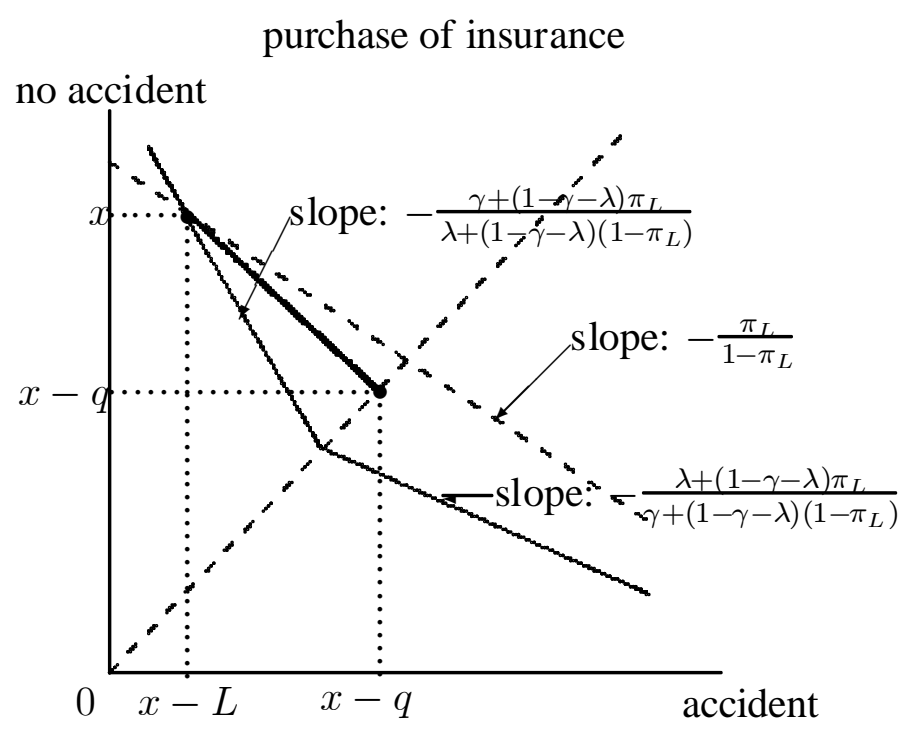

Figure 4: Insurance

Having purchased the insurance, the difference in Choquet expected utilities between buying the lottery ticket and not may now be expressed as

$$
\begin{aligned}
& {\left[\lambda+(1-\gamma-\lambda) \cdot \pi_{W}\right] \cdot u(x+W-p-q)} \\
& +\left[\gamma+(1-\gamma-\lambda) \cdot\left(1-\pi_{W}\right)\right] \cdot u(x-p-q)-u(x-q)
\end{aligned}
$$




$$
\begin{aligned}
& >\lambda \cdot u(x+W-p-q)+(1-\lambda) \cdot u(x-p-q)-u(x-q) \\
& \geq \lambda \cdot[u(x+W-p-q)-u(x-q)]-p \cdot u^{\prime}(x-q) .
\end{aligned}
$$

The last inequality follows from the concavity of $u$. For $\lambda>0$ and $u$ strictly increasing, there is a lottery $\left(W, \pi_{W}, p\right)$ with $W$ high enough and $\pi_{W}$ small enough such that $\pi_{W} \cdot W<$ $p$ and

$$
\frac{\lambda \cdot[u(x+W-p-q)-u(x-q)]}{p}>u^{\prime}(x-q) .
$$

Notice that this is true for any degree of concavity of $u$. Optimism makes lotteries with high prizes and low probabilities of winning attractive even for individuals who are averse to accepting actuarially fair fifty-fifty gambles. Figure 5 illustrates the desirability of the purchase of an unfair lottery ticket for the case where $u$ is affine.

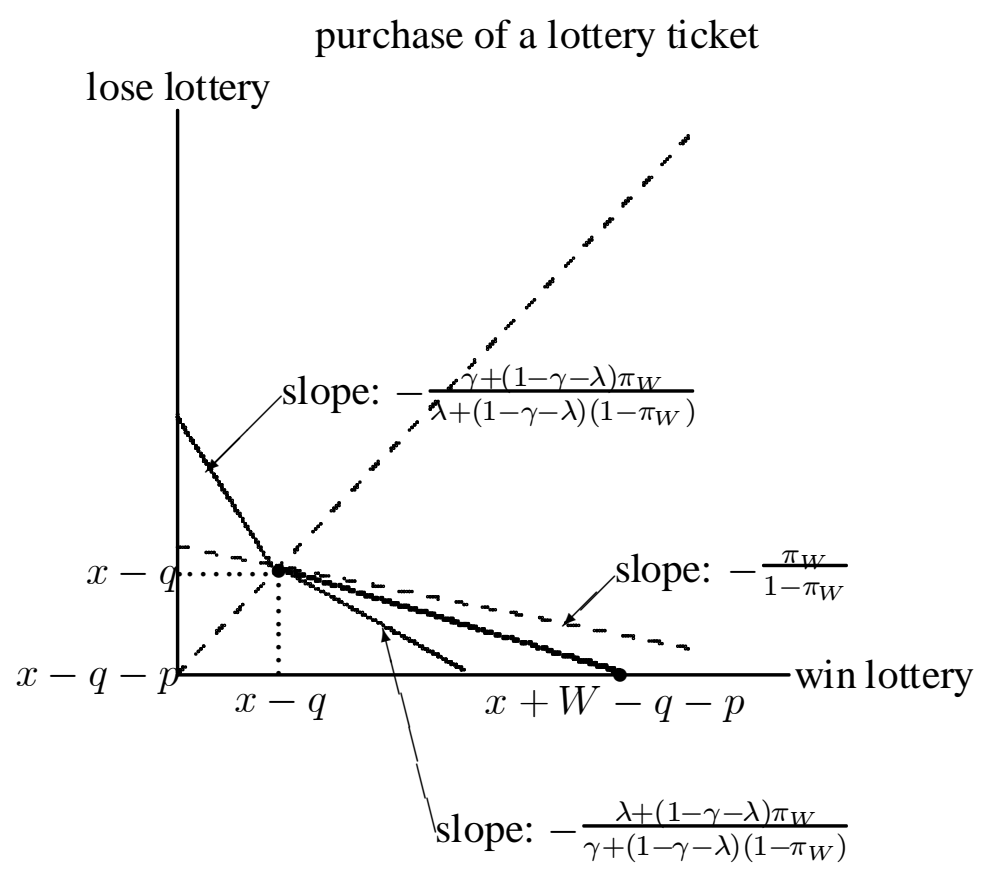

Figure 5: Gambling

\subsection{Portfolio choice}

There are numerous puzzles in portfolio choice theory. THALER (2000) provides a 
stimulating exposition of some well-known irregularities. These puzzles highlight inconsistencies between standard economic theories and empirical regularities. Naturally, not all can be related to optimism or pessimism. The following two puzzles however can be explained easily by a small degree of optimism and pessimism.

The equity premium puzzle refers to the large difference between the average return on a stock portfolio and the return of a fixed interest bearing bond which was first noted by Mehra \& PRESCOTT (1985). The implied risk premium appears to be too big to be explained by risk aversion as modelled by a concave von Neumann-Morgenstern utility function. The conservative behavior in the face of uncertainty suggested by such a high risk premium stands in stark contrast to the observation that small firms with high-risk stocks seem to attract investors' interest more than is warranted by their average returns. To invest in stock of young "promising" companies appears to be extraordinarily risky. Yet such uncertainty did not deter investors who otherwise requested a surprisingly high risk premium.

We study a simple financial market system with a representative investor, one risky and one riskless as set and an exogenous supply of assets. This framework suffices to illustrate the impact of optimism and pessimism on portfolio choice. With well-known modifications these results carry over to more general models of financial markets.

Consider an investor with initial wealth $W_{0}$ who can invest in two assets, a stock with uncertain returns and a bond with a certain payoff. The following table summarizes the notation of the assets.

\begin{tabular}{c|c|c|c|} 
asset & quantity & price & payoff in state $s \in S$ \\
\hline stock & $a$ & $q$ & $r_{s}$ \\
\hline bond & $b$ & 1 & $r$ \\
\hline
\end{tabular}

Preferences of the investor are represented by a Choquet expected utility $V\left(W_{1}, \ldots, W_{S}\right)$ of end-of-period wealth, $W_{s}=r_{s} \cdot a+r \cdot b$, with respect to a neo-additive capacity

$$
\begin{aligned}
& V\left(W_{1}, \ldots, W_{S}\right) \\
& :=\gamma \cdot \min \left\{u\left(W_{1}\right), \ldots, u\left(W_{S}\right)\right\}+\lambda \cdot \max \left\{u\left(W_{1}\right), \ldots, u\left(W_{S}\right)\right\} \\
& +(1-\gamma-\lambda) \cdot \sum_{s \in S} \pi_{s} \cdot u\left(W_{s}\right) .
\end{aligned}
$$

Using the budget constraint, $W_{0}=q \cdot a+b$, to substitute for the bond, one gets wealth 
as a function of stock transactions $a$,

$$
W_{s}=r \cdot W_{0}+\left[r_{s}-q \cdot r\right] \cdot a .
$$

Denoting by $\bar{r}=\max \left\{r_{1}, \ldots, r_{S}\right\}$ and $\underline{r}=\min \left\{r_{1}, \ldots, r_{S}\right\}$ the maximal and minimal returns of the risky stock, one can write the Choquet expected utility from a stock investment $a>0$ as

$$
\begin{aligned}
V(a):=\quad \gamma & \cdot u\left(r \cdot W_{0}+[\underline{r}-q \cdot r] \cdot a\right) \\
& +\lambda \cdot u\left(r \cdot W_{0}+[\bar{r}-q \cdot r] \cdot a\right) \\
& +(1-\gamma-\lambda) \cdot \sum_{s \in S} \pi_{s} \cdot u\left(r \cdot W_{0}+\left[r_{s}-q \cdot r\right] \cdot a\right)
\end{aligned}
$$

For a stock market equilibrium price $q^{*}$ with an aggregate endowment of equity $A>0$ and bonds $B=0$ where the single investor maximizes Choquet expected utility $V(a)$,

$$
\begin{aligned}
V^{\prime}(A)= & \gamma \cdot u^{\prime}\left(r \cdot W_{0}+\left[\underline{r}-q^{*} \cdot r\right] \cdot A\right) \cdot\left[\underline{r}-q^{*} \cdot r\right] \\
& +\lambda \cdot u^{\prime}\left(r \cdot W_{0}+\left[\bar{r}-q^{*} \cdot r\right] \cdot A\right) \cdot\left[\bar{r}-q^{*} \cdot r\right] \\
& +(1-\gamma-\lambda) \cdot \sum_{s \in S} \pi_{s} \cdot u^{\prime}\left(r \cdot W_{0}+\left[r_{s}-q^{*} \cdot r\right] \cdot A\right) \cdot\left[r_{s}-q^{*} \cdot r\right]=0
\end{aligned}
$$

must hold in equilibrium. Substituting for the initial wealth $W_{0}=q^{*} \cdot A$, this equilibrium condition can be solved explicitly for the equilibrium stock price $q^{*}$,

$$
q^{*}=\frac{\gamma \cdot u^{\prime}(\underline{r} \cdot A) \cdot \underline{r}+\lambda \cdot u^{\prime}(\bar{r} \cdot A) \cdot \bar{r}+(1-\gamma-\lambda) \cdot \sum_{s \in S} \pi_{s} \cdot u^{\prime}\left(r_{s} \cdot A\right) \cdot r_{s}}{r \cdot\left[\gamma \cdot u^{\prime}(\underline{r} \cdot A)+\lambda \cdot u^{\prime}(\bar{r} \cdot A)+(1-\gamma-\lambda) \cdot \sum_{s \in S} \pi_{s} \cdot u^{\prime}\left(r_{s} \cdot A\right)\right]} .
$$

The case of subjective expected utility, $\gamma=\lambda=0$, is the reference situation against which we can assess the impact of optimism or pessimism. Denote by $q_{0}^{*}$ the equity price in this case,

$$
q_{0}^{*}=\frac{\sum_{s \in S} \pi_{s} \cdot u^{\prime}\left(r_{s} \cdot A\right) \cdot r_{s}}{r \cdot \sum_{s \in S} \pi_{s} \cdot u^{\prime}\left(r_{s} \cdot A\right)} .
$$

The equity premium is defined as the ratio

$$
\alpha\left(q^{*}\right):=\frac{\sum_{s \in S} \pi_{s} \cdot r_{s}}{q^{*} \cdot r} .
$$

The smaller $q^{*}$ the greater the equity premium.

Consider first the case of a risk-neutral investor, $u^{\prime}(\cdot)=k$. In this case, the equilibrium stock price equals the discounted expected return of the stock plus an optimism and pes- 
simism related premium

$$
\begin{aligned}
q^{*} & =\frac{\sum_{s \in S} \pi_{s} \cdot r_{s}}{r}+\frac{\gamma \cdot\left(r-\sum_{s \in S} \pi_{s} \cdot r_{s}\right)+\lambda \cdot\left(\bar{r}-\sum_{s \in S} \pi_{s} \cdot r_{s}\right)}{r} \\
& =q_{0}^{*}+\frac{1}{r} \cdot\left[\gamma \cdot\left(\underline{r}-\mathcal{E}_{\pi} r_{s}\right)+\lambda \cdot\left(\bar{r}-\mathcal{E}_{\pi} r_{s}\right)\right],
\end{aligned}
$$

where we denote the expected return of the stock by $\mathcal{E}_{\pi} r_{s}:=\sum_{s \in S} \pi_{s} \cdot r_{s}$.

Since $\bar{r}>\mathcal{E}_{\pi} r_{s}>\underline{r}$, optimism will add a positive premium, $\lambda \cdot\left(\bar{r}-\mathcal{E}_{\pi} r_{s}\right)$, to the reference price $q_{0}^{*}$, while pessimism will make the premium negative, $\gamma \cdot\left(\underline{r}-\mathcal{E}_{\pi} r_{s}\right)$. For a riskneutral investor, we can note that

- the equity premium will be the higher the more pessimistic the investor is, i.e. the smaller $\gamma$;

- if there is no optimism, $\lambda=0$, then the equity premium required by a pessimistic investor will be strictly higher than the one based on the subjective probability distribution $\pi$ alone, $\alpha\left(q^{*}\right)>\alpha\left(q_{0}^{*}\right)$;

- if both optimism and pessimism prevail, but $\gamma>\lambda$ as in most experimental studies, then a sufficient condition for an equity premium $\alpha\left(q^{*}\right)$ exceeding $\alpha\left(q_{0}^{*}\right)$ is an average return exceeding the average of the minimum and the maximum return,

$$
\frac{\bar{r}+\underline{r}}{2}<\mathcal{E}_{\pi} r_{s}
$$

For a risk-averse investor with a strictly decreasing marginal utility function $u^{\prime}(\cdot)$ similar results can be deduced which we summarize in a lemma.

Lemma 4.1 $A$ risk-averse and pessimistic investor, i.e., with $\lambda=0, \gamma>0, u^{\prime}(\cdot)$ strictly decreasing, requires an equity premium exceeding the equity premium with risk but no pessimism,

$$
\alpha\left(q^{*}\right)>\alpha\left(q_{0}^{*}\right)
$$

\section{Proof. Appendix.}

In recent years "new stock markets" have emerged in many developed countries where stock of start-up firms is traded. These markets were opened in order to provide ven- 
ture capital for new high-risk enterprises with great potential. In the light of the rather conservative behavior reflected in the equity premium puzzle it is even more surprising that investors were willing to bet substantial amounts of wealth on firms with no record of earnings.

Optimism and pessimism as modelled with a neo-additive capacity enables us to explain such behavior. In fact, we can show that for an arbitrary small degree of optimism there are maximal returns of a firm high enough to induce a positive stock price for high-risk firms with potentially high returns. Reconsider the stock market equilibrium price of Equation (*) and assume, without loss of generality, that the firm's stock pays off a return $R$ only in state 1 . Hence, $\bar{r}=r_{1}=R$ and $\underline{r}=r_{s}=0$ for all $s \neq 1$. Suppose the expected return of the firm is bounded away from zero, $\pi_{1} \cdot R \geq \kappa>0$. Then the equilibrium price satisfies

$$
\begin{aligned}
q^{*} & =\frac{1}{r} \cdot \frac{\lambda \cdot u^{\prime}(R \cdot A) \cdot R+(1-\gamma-\lambda) \cdot \pi_{1} \cdot u^{\prime}(R \cdot A) \cdot R}{\gamma \cdot u^{\prime}(0)+\lambda \cdot u^{\prime}(R \cdot A)+(1-\gamma-\lambda) \cdot\left[\pi_{1} \cdot u^{\prime}(R \cdot A)+\sum_{s \neq 1} \pi_{s} \cdot u^{\prime}(0)\right]} \\
& =\frac{R}{r} \cdot \frac{u^{\prime}(R \cdot A) \cdot\left[\lambda+(1-\gamma-\lambda) \cdot \pi_{1}\right]}{u^{\prime}(R \cdot A) \cdot\left[\lambda+(1-\gamma-\lambda) \cdot \pi_{1}\right]+u^{\prime}(0) \cdot\left[\gamma \cdot+(1-\gamma-\lambda) \cdot\left(1-\pi_{1}\right)\right]} \\
& \geq \frac{R}{r} \cdot\left[\lambda+(1-\gamma-\lambda) \cdot \pi_{1}\right] \\
& >\frac{R}{r} \cdot \lambda,
\end{aligned}
$$

where the first inequality follows from $u^{\prime}(0) \geq u^{\prime}(R \cdot A)$ and the second strict inequality from the positive expected return.

It is clear that with some optimism, $\lambda>0$, even a vanishing probability of success $\pi_{1}$ will not deter investors provided the return rises sufficiently, $R \geq \kappa / \pi_{1}$. The stock market price will not collapse. There is no contradiction if investors buy high-risk stock because of optimism, $\lambda>0$, and require an "excessive" equity premium. Adam Smith's observation that even "sober people" do play lotteries and Robert Shiller's observed "exuberance" in the stock market can be reconciled with rational decision making under uncertainty, if one allows for optimism and pessimism as modelled by neo-additive capacities. 


\section{Behavioral axioms}

We present our theory in the context of a variant of SAVAGE'S (1954) purely subjective uncertainty framework employed by GHIRARDATO \& MARINACCI (2001) and GHIRardato, Maccherioni, MarinacCi \& Siniscalchi (2002) (hereafter, GMMS). The state space $S$ is taken to be the same as was defined in section 2 above. Let $X$, the set of outcomes, be a connected and separable topological space. An act is a function (measurable with respect to $\mathcal{E}$ ) $f: S \rightarrow X$ with finite range, $\mathcal{F}$ denotes the set of such acts and is endowed with the product topology induced by the topology on $X$. We shall identify each $x \in X$ with the constant act, $f(s)=x$ for all $s \in S$. For any pair of acts $f, g$ in $\mathcal{F}$ and any event $E \in \mathcal{E}, f_{E} g$ will denote the act $h \in \mathcal{F}$, formed from the concatenation of the two acts $f$ and $g$, in which $h(s)$ equals $f(s)$ if $s \in E$, and equals $g(s)$ if $s \notin E$. Let $\succsim$ denote the individual's preference relation on $\mathcal{F}$. For any $f \in \mathcal{F}$, the certainty equivalent of $f$, denoted by $m(f)$, is the set of constant acts that are indifferent to $f$. That is, $x \in m(f)$, if $x \sim f$. Although many constant acts may be equivalent, when there is no risk of confusion, we shall write $m(f)$ to indicate an arbitrary member of the set. We say $f$ and $g$ are comonotonic if for every pair of states $s$ and $s^{\prime}$ in $S, f(s) \succ f\left(s^{\prime}\right)$ implies $g(s) \succsim g\left(s^{\prime}\right)$.

Adopting GHIRARDATO AND MARINACCI's (2001) nomenclature, we say an event $E \in \mathcal{E}$ is null (respectively, universal) for a preference relation $\succsim$, if $x_{E} y \sim y$ (respectively, $x_{E} y \sim x$ ) for all outcomes $x, y \in X$, such that $x \succ y{ }^{4}$ An event $E \in \mathcal{E}$, is essential for $\succsim$ if for some $x, y \in X$ we have $x \succ x_{E} y \succ y$. Let $\mathcal{N}$ (respectively, $\mathcal{U}$ and $\mathcal{E}^{*}$ ) denote the set of null (respectively, universal and essential) events.

For ease of exposition and without any essential loss of generality we assume there exist outcomes 0 and $M$ in $X$, that are, respectively, the "best" and "worst" outcomes in $X$, in the sense that $M \succ 0$ and $M \succsim x \succsim 0$ for all $x \in X$.

Neo-additive capacities are a special case of the Choquet expected utility theory. In order to obtain a behavioral characterization, we seek to modify the axioms of GMMS appro-

$\overline{4}$ This is weaker than Savage's original notion of 'null' event which requires $f_{E} g \sim g$ for all $f, g \in \mathcal{F}$. 
priately. Their key innovation is to define a behavioral definition of 'subjective mixtures' of acts which allows them to define in a Savage framework of purely subjective uncertainty, analogs to axioms based on probability mixtures that play such a key role in the Anscombe-Aumann framework.

The first axiom requires the preference order to be a complete preorder.

\section{Axiom 1 (Ordering)}

The preference relation $\succsim$ on $\mathcal{F}$ is complete, reflexive and transitive.

The neo-additive expected utility representation allows for the discontinuous over-weighting of events on which extreme, i.e. either best or the worst, outcomes obtain. Hence, standard continuity with respect to the product topology cannot be expected to hold for the whole preference relation. Following Ghirardato \& MARINACCI (2001) we only require a weaker notion of pointwise convergence, where in this product topology, we say a net $\left\{f_{\alpha}\right\}_{\alpha \in D} \subseteq \mathcal{F}$ converges pointwise to $f \in \mathcal{F}$, if and only if $f_{\alpha}(s) \rightarrow f(s)$ for all $s \in S$.

\section{Axiom 2 (Continuity).}

Let $\left\{f_{\alpha}\right\}_{\alpha \in D} \subseteq \mathcal{F}$ be a net that converges pointwise to $f$ and such that all $f_{\alpha}$ s and $f$ are measurable with respect to the same finite partition.. If $f_{\alpha} \succsim g$ (respectively, $g \succsim f_{\alpha}$ ) for all $\alpha \in D$, then $f \succsim g$ (respectively, $g \succsim f$ ).

We also adopt the monotonicity axiom of CHEW \& KARNI (1994) which combines statewise dominance with a weakening of Savage's axiom P3.

\section{Axiom 3 (Eventwise Monotonicity).}

For any pair of acts, $f, g \in \mathcal{F}$, if $f(s) \succsim g(s)$ for all $s \in S$, then $f \succsim g$.

In addition, for any triple of outcomes $x, y, z \in X$, and any event $E \in \mathcal{E}$

(a) if $E \notin \mathcal{N}$ and $x \succsim z, y \succsim z$ then $x \succ y \Rightarrow x_{E} z \succ y_{E} z$;

(b) if $E \notin \mathcal{U}$ and $z \succsim x, z \succsim y$ then $x \succ y \Rightarrow z_{E} x \succ z_{E} y$.

As Ghirardato \& Marinacci (2001) note, Axioms 1 and 3 imply that the sets of null, universal and essential events form a partition of $\mathcal{E}$.

The next axiom due to Ghirardato \& MarinacCi (2001) builds on the idea of 
NAKamura (1990) and Gul (1992) of a 'subjective mixture' of two acts $f$ and $g$. Fix some event $E$, and then construct state by state an act which yields at each state $s$, the certainty equivalent of the bet $f(s)_{E} g(s)$. Formally, the statewise (event) $E$-mixture of $f$ and $g$, denoted as $f^{E} g$, is taken to be the act

$$
f^{E} g(s)=m\left(f(s)_{E} g(s)\right) .
$$

Adopting the shorthand $\{x, y\} \succsim z$ for $x \succsim z$ and $y \succsim z$, and $z \succsim\{x, y\}$ for $z \succsim x$ and $z \succsim x$, the next axiom may be stated as follows.

\section{Axiom 4 (Binary Comonotonic Act Independence)}

For any event $A \in \mathcal{E}^{*}$, any event $B \in \mathcal{E}$, and for all $f, g, h \in \mathcal{F}$, such that $f=x_{A} y, g=$ $x_{A}^{\prime} y^{\prime}, h=x_{A}^{\prime \prime} y^{\prime \prime}$. If $f, g, h$ are pairwise comonotonic, and $\left\{x, x^{\prime}\right\} \succsim x^{\prime \prime}$ and $\left\{y, y^{\prime}\right\} \succsim y^{\prime \prime}$ (or $x^{\prime \prime} \succsim\left\{x, x^{\prime}\right\}$ and $y^{\prime \prime} \succsim\left\{y, y^{\prime}\right\}$ ), then

$$
f \succsim g \Rightarrow f^{B} h \succsim g^{B} h
$$

As its names suggests, Binary Comontonic Act Independence, means that the preference relation restricted to acts that are measurable with respect to two-element partitions, conforms to the theory of Choquet Expected Utility. With these four axioms, GHIRARDATO \& MARINACCI (2001) were able to prove that the preference relation admits what they dubbed a (canonical) biseparable representation, namely, a Choquet Expected Utility representation defined on this restricted set of acts.

Proposition 5.1 (Ghirardato and Marinacci [2001, Theorem 11]) Let $X$ be a connected and separable topological space and let $\succsim$ be a binary relation on $\mathcal{F}$ for which there exist outcomes 0 and $M$ in $X$, such that $M \succ 0$ and $M \succsim x \succsim 0$ for all $x \in X$. Then the following are equivalent:

(i) $\succsim$ satisfies Axioms 1-4 and there exists an essential event $A$.

(ii) There exists a continuous utility index $u: X \rightarrow[0,1]$ and a capacity $\nu: \mathcal{E} \rightarrow[0,1]$ such that for all $x, y, x^{\prime}, y^{\prime}$, such that $x \succsim y$ and $x^{\prime} \succsim y^{\prime}$ and all $E, E^{\prime} \in \mathcal{E}$

$$
x_{E} y \succsim x_{E^{\prime}}^{\prime} y^{\prime} \Leftrightarrow \nu(E) u(x)+(1-\nu(E)) u(y) \geq \nu\left(E^{\prime}\right) u\left(x^{\prime}\right)+\left(1-\nu\left(E^{\prime}\right)\right) u\left(y^{\prime}\right) .
$$


Furthermore, if the set of essential events $\mathcal{E}^{*}$ is non-empty then the capacity in (2) is unique and the utility index in (2) is unique up to positive affine transformations.

Proposition 5.1 implies that events $E \in \mathcal{E}$ are null if and only if $\nu(E)=0$, universal if and only if $\nu(E)=1$ and essential if and only if $0<\nu(E)<1$ holds.

It remains to impose an appropriate version of an independence-type axiom that extends the biseparable CEU representation obtained in Proposition 5.1 to the whole domain $\mathcal{F}$ and moreover entails that the capacity in that representation is neo-additive.

To do this, we first need to define GMMS's notion of a 'subjective mixture' of two acts. We begin with their definition of a 'preference average' of two consequences..

Definition 5.1 Fix $x, y \in X$, such that $x \succ y$. We say that a consequence $z \in X$ is a preference average of $x$ and $y$ (given $E$ ) if $x \succsim z \succsim y$ and

$$
x_{E} y \sim m\left(x_{E} z\right)_{E} m\left(z_{E} y\right)
$$

Ghirardato \& MarinacCi (2001) show that our Axioms A1 to A4 together with the connectedness of $X$ imply that the preference average in Definition 5.1 is well-defined. The reason for their nomenclature becomes apparent if we consider for a preference relation that satisfies Axioms 1-4, the preference average of $x$ and $y$ given an essential event $E$. From Proposition (5.1) we obtain the equality

$$
\begin{aligned}
& \nu(E) u(x)+(1-\nu(E)) u(y) \\
= & \nu(E) u\left(m\left(x_{E} z\right)\right)+(1-\nu(E)) u\left(m\left(z_{E} y\right)\right) \\
= & {[\nu(E)]^{2} u(x)+2 \nu(E)(1-\nu(E)) u(z)+(1-\nu(E))^{2} u(y) . }
\end{aligned}
$$

Notice that if the event $E$ is essential then $0<\nu(E)<1$, and so solving for $u(z)$ yields

$$
u(z)=\frac{1}{2} u(x)+\frac{1}{2} u(y),
$$

which is independent of $E$. We shall therefore denote by $(1 / 2) x \oplus(1 / 2) y$ the preference average of the outcomes $x$ and $y$. To deliver weighted averages of $x$ and $y$, we follow the line of argument detailed in GMMS. That is, by using iterated averages (for example, $(1 / 2) x \oplus((1 / 2) x \oplus(1 / 2) y)$ corresponds to a $(3 / 4,1 / 4)$ - weighted average of $x$ and 
$y$ ) and appealing to standard continuity arguments, it is possible to identify, for any $\alpha$ in $[0,1]$ and every $x$ and $y$ in $X$, the weighted preference averages characterized by

$$
u(z)=\alpha u(x)+(1-\alpha) u(y) .
$$

With slight abuse of notation, we shall let $\alpha x \oplus(1-\alpha) y$ (or, equivalently, $(1-\alpha) y \oplus$ $\alpha x$ ) denote an arbitrary element of the indifferent set of outcomes for such preference averages. We are now in a position to define subjective mixtures of acts.

Definition 5.2 Fix $f, g \in \mathcal{F}$ and $\alpha \in[0,1]$. A subjective mixture of $f$ and $g$ with weight $\alpha$ is any act $h \in \mathcal{F}$ such that $h(s) \sim \alpha f(s) \oplus(1-\alpha) g(s)$ for each $s \in S$.

As GMMS note, all subjective mixtures of $f$ and $g$ with weight $\alpha$ are state-wise indifferent, and hence by Axiom 3 (i), indifferent. So we follow them and denote by $\alpha f \oplus(1-\alpha) g$ any one of them.

Our final axiom is key to characterizing the decision maker's attitudes towards events that yield extreme outcomes. We first need, however, to define for each act which events the decision maker views as yielding the extreme outcomes. We begin with preference-based definitions for the infimum and the supremum of an act.

Definition 5.3 Fix $f \in \mathcal{F}$. An outcome $z \in X$ is said to be in the indifference set of the infimum of $f, z \in \inf _{\succeq}(f)$, if for $A:=f^{-1}(x: z \succ x), z_{A} f \sim f$ and if for every $y \succ z$ and $B:=f^{-1}(x: y \succeq x), y_{B} f \succ f$. Similarly, an outcome $z \in X$ is said to be in the indifference set of the supremum of $f, z \in \sup _{\succeq}(f)$ iffor $A:=f^{-1}(x: x \succ z), z_{A} f \sim f$ and iffor every $y$ such that $z \succ y$ and $B:=f^{-1}(x: x \succeq y), f \succ y_{B} f$.

Although $\inf _{\succeq}(f)$ and $\sup _{\succeq}(f)$ are defined to be indifference sets of outcomes, when there is no risk of confusion, we shall write $\inf _{\succeq}(f)$ and $\sup _{\succeq}(f)$ to indicate arbitary members of these respective sets.

From the definition of a subjective mixture and equation (3) it follows that for every $f, g$ $\in \mathcal{F}, \alpha \in[0,1]$ and $s \in S$

$$
u(\alpha f(s) \oplus(1-\alpha) g(s))=\alpha u(f(s))+(1-\alpha) u(g(s)) .
$$


Hence, if there is a non-null event on which both acts $f$ and $g$ attain their supremum, that is, the set $E=\left\{s \in S: f(s) \in \sup _{\succeq}(f)\right\} \cap\left\{s \in S: g(s) \in \sup _{\succeq}(g)\right\}$ is not null, then a subjective mixture of these two acts will also attain its supremum on this set. That is, for any $\alpha$ in $(0,1), \alpha f \oplus(1-\alpha) g(s) \in \sup _{\succeq}(\alpha f \oplus(1-\alpha) g)$, for all $s \in E$. On the other hand, if the set $E$ is null, then a subjective mixture of these acts need not necessarily attain its supremum on any state in $\left\{s \in S: f(s) \in \sup _{\succeq}(f)\right\}$ nor on any state in $\left\{s \in S: g(s) \in \sup _{\succeq}(g)\right\}$. In this case, the supremum attained will be less than that which would have been attained if there had been a common non-null event on which both acts attained their supremum. Similarly, if the set $F=\left\{s \in S: f(s) \succ \inf _{\succeq}(f)\right\} \cup$ $\left\{s \in S: g(s) \succ \inf _{\succeq}(g)\right\}$ is not universal, then for any $\alpha \in(0,1), \alpha f \oplus(1-\alpha) g(s)$ $\in \inf _{\succeq}(\alpha f \oplus(1-\alpha) g)$, for all $s \notin F$. While the infimum attained for that subjective mixture would be no smaller if the set $F$ were universal.

The idea behind the next axiom is that if the decision maker is sensitive about extreme events, both for good and bad outcomes, then subjective mixtures of acts for which supremum outcomes are obtained on common non-null events will be viewed relatively favorably while subjective mixtures of acts for which infimum outcomes are obtained on a common event whose complement is not universal will be viewed relatively unfavorably.

To facilitate the formulation of this final axiom, define an act $h$ to be a member of $\mathcal{F}(f)$, if the set of states on which a non-infimum outcome obtains either for $h$ or for $f$ is not universal. That is,

$$
\mathcal{F}(f):=\left\{h \in \mathcal{F} \mid\left\{s \in S: f(s) \succ \inf _{\succeq}(f)\right\} \cap\left\{s \in S: g(s) \succ \inf _{\succeq}(g)\right\} \notin \mathcal{U}\right\} .
$$

Similarly, denote by $\overline{\mathcal{F}}(f)$ the set of acts with some maximum outcomes on a common non-null event as the act $f$. That is,

$$
\overline{\mathcal{F}}(f):=\left\{h \in \mathcal{F} \mid\left\{s \in S: f(s) \in \sup _{\succeq}(f)\right\} \cap\left\{s \in S: g(s) \in \sup _{\succeq}(g)\right\} \notin \mathcal{N}\right\}
$$

In GMMS's axiomatization of Choquet Expected Utility, their key axiom is the restriction of an independence type axiom to subjective mixtures of co-monotonic acts. The 
Choquet integral of a neo-additive capacity satisfies the independence axiom for all acts with the best and worst outcomes on the same events, respectively. Hence, we effectively strengthen their co-monotonic independence axiom by requiring it to hold for all acts which obtain their supremum (and, respectively, their infimum) on some common non-null event in common.

\section{Axiom 5 (Extreme Events Sensitivity)}

For any $f, g, h \in \mathcal{F}$ such that $f \sim g$ and $h \in \underline{\mathcal{F}}(g) \cap \overline{\mathcal{F}}(g)$, and any $\alpha \in(0,1]$,

1. If $h \in \underline{\underline{\mathcal{F}}}(f)$ then $\alpha g \oplus(1-\alpha) h \succsim \alpha f \oplus(1-\alpha) h$,

2. If $h \in \overline{\overline{\mathcal{F}}}(f)$ then $\alpha f \oplus(1-\alpha) h \succsim \alpha g \oplus(1-\alpha) h$.

Acts that are comonotonic with $f$ are elements of $\mathcal{F}(f) \cap \overline{\mathcal{F}}(f)$. Hence Axiom 5 implies comonotonic independence for the indifference relation. In the proof of our representation result, we show that in conjunction with the other axioms it characterizes a subclass of the family of Choquet expected utility functionals.

Theorem 5.1 Let $X$ be a connected and separable topological space, let $\mathcal{E}^{*}$ contain at least four elements $E_{1}, E_{2}, E_{3}$ and $E_{4}$ that together form a partition of $S$, and let $\succsim$ be a binary relation on $\mathcal{F}$ for which there exist outcomes 0 and $M$ in $X$, such that $M \succ 0$ and $M \succsim x \succsim 0$ for all $x \in X$. The following two statements are equivalent:

1. The preference relation $\succsim$ on $\mathcal{F}$ satisfies Ordering, Continuity, Eventwise Monotonicity, Binary Comonotonic Act Independence and Extreme Events Sensitivity.

2. There exists a unique neo-additive capacity $\nu$ on $\mathcal{E}$ and a continuous real-valued function $u$ on $X$, unique up to positive affine transformations, such that for all $f, g \in \mathcal{F}$

$$
f \succsim g \Leftrightarrow \int u \circ f d \nu \geq \int u \circ g d \nu
$$

Proof. Appendix.

Remark: Comparing the conditions of Proposition 3.1 and Theorem 5.1, one may wonder 
why the set of possible events $\mathcal{E}^{*}$ was required to have at least three non-intersecting elements but had to have at least four such events in the latter. In Proposition 3.1 three such events are required to make statement (ii a) meaningful but this statement had not to be derived. In Theorem 5.1 four non-null and non-universal events are necessary in order to prove that the capacity $\nu$ which we deduce satisfies statement (ii a) of Proposition 3.1. In order to see that four states are necessary for $\nu$ to satisfy statement (ii a), consider the following counterexample. The capacity $\nu$ on $S=\left\{s_{1}, s_{2}, s_{3}\right\}$ defined by

$$
\begin{aligned}
& \nu\left(s_{1}\right)=\frac{1}{2}, \quad \nu\left(s_{2}\right)=\frac{1}{3}, \quad \nu\left(s_{3}\right)=\frac{1}{4}, \\
& \nu\left(\left\{s_{1}, s_{2}\right\}\right)=\nu\left(\left\{s_{1}, s_{3}\right\}\right)=\nu\left(\left\{s_{2}, s_{3}\right\}\right)=\frac{1}{2}
\end{aligned}
$$

satisfies statements (ii b) and (ii c) but not (ii a) of Proposition 3.1 Hence, it is not neoadditive.

\section{Concluding remarks}

Optimism and pessimism have long been recognized as important determinants of economic behavior. Subjective expected utility theory assumes that the impact of uncertainty can be reduced to the statistical properties of a probability distribution. This reduction extends economic analysis to situations under uncertainty where one could rightfully neglect psychological aspects relating to the focal attraction of the best and worst outcome of economic choices.

In this paper we have introduced a special case of capacity and its Choquet integral which captures aspects of optimism and pessimism without abandoning the subjectively probabilistic approach all together. In particular, subjective expected utility is always contained as a special parametric case in this approach. Moreover, as in EICHBERGER \& KeLSEY (2000), the additive part of a neo-additive capacity can be determined endogenously in equilibrium.

Most importantly neo-additive capacities open new avenues of research. It appears natural to view the degree of confidence which a decision maker holds in a probabilistic assessment of an uncertain situation as dependent on past experience and subject to inf lu- 
ence from other people's beliefs. Optimism and pessimism may spread in a population. Attitudes towards uncertain outcomes may be contagious leading to general swings in optimism and pessimism. So "irrational exuberance" as observed by SHILLER (2001) may become amenable to formal economic analysis after all. 


\section{Appendix A: Proofs}

For notational convenience, let $\widehat{\mathcal{E}}:=\{E \in \mathcal{E} \mid 0<\nu(E)<1\}$.

The following preliminary lemma establishes uniqueness of $\gamma, \lambda$, and $\pi$.

Lemma: Let $\nu$ be a capacity on $(S, \mathcal{E})$, where $\widehat{\mathcal{E}}$ contains at least three elements $E_{1}, E_{2}$, and $E_{3}$, such that $E_{i} \cap E_{j}=\varnothing$, for all $i \neq j$. Then $(\gamma, \lambda, \pi)$ is unique.

Proof. Let $(\gamma, \lambda, \pi)$ be a given vector of parameters of $\nu$. Denote by $\alpha:=1-\gamma-\lambda$ and $\widetilde{\pi}(E):=\alpha \cdot \pi(E)$ for all $E \in \mathcal{E}$.

For any $E, F \in \widehat{\mathcal{E}}$ such that $E \cap F=\emptyset$ and $E \cup F \in \widehat{\mathcal{E}}, \widetilde{\pi}(E)$ is uniquely defined by $\tilde{\pi}(E):=\nu(E \cup F)-\nu(F)$.

Assume now that $E \in \widehat{\mathcal{E}}$, then there exists $\left(E_{1}, E_{2}\right) \in \widehat{\mathcal{E}} \times \widehat{\mathcal{E}}$ such that $E_{1} \cap E_{2}=\emptyset$ and $E_{1} \cup E_{2}=E$. Hence, $\widetilde{\pi}(E)$ is uniquely defined by $\widetilde{\pi}(E)=\widetilde{\pi}\left(E_{1}\right)+\widetilde{\pi}\left(E_{2}\right)$.

This implies that $\widetilde{\pi}(E)$ is uniquely defined for all $E \in \widehat{\mathcal{E}}$ and that $\lambda$ is uniquely defined by $\lambda:=\nu(E)-\widetilde{\pi}(E)$ for all $E \in \widehat{\mathcal{E}}$.

Let $\left\{E_{i} \in \widehat{\mathcal{E}} \mid i=1, . ., 3\right\}$ be a partition of $S$. Then $\alpha$ is uniquely defined by $\alpha:=\sum_{i=1}^{3} \widetilde{\pi}\left(E_{i}\right)$. Hence, $\gamma$ is unique. Finally, either $\alpha=0$ and there is no $\pi$ in the expression of $\nu$, or $\alpha>0$ and $\pi$ is uniquely defined for any $E \in \widehat{\mathcal{E}}$ by $\pi(E):=\frac{\widetilde{\pi}(E)}{\alpha}$.

\section{A.1 Proof of Proposition 3.1}

(i) $\Longrightarrow$ (ii). This follows from the definition of a neo-additive capacity.

(ii) $\Longrightarrow$ (i).

(a) First we define a non-negative simple additive measure $\widetilde{\pi}$ on $\widehat{\mathcal{E}}$.

Case 1: $E \in \widetilde{\mathcal{E}}:=\{E \in \widehat{\mathcal{E}} \mid \exists F \in \widehat{\mathcal{E}}, E \cap F=\emptyset, E \cup F \in \widehat{\mathcal{E}}\}$.

Define $\widetilde{\pi}(E):=\nu(E \cup F)-\nu(F)$ for all $F \in \widehat{\mathcal{E}}$ such that $E \cap F=\emptyset, E \cup F \in \widehat{\mathcal{E}}$. Property (a) implies that $\widetilde{\pi}(E)$ is well-defined.

Case 2: $E \in \widehat{\mathcal{E}}-\widetilde{\mathcal{E}}$. Hence $F=S-E \notin \widetilde{\mathcal{E}}$ and there exists a finite partition of $E$, 
$\left\{E_{i} \in \widetilde{\mathcal{E}} \mid i=1, \ldots, n\right\}$. Define $\widetilde{\pi}(E):=\sum_{i=1}^{n} \widetilde{\pi}\left(E_{i}\right)$. It is well defined because

$$
\begin{aligned}
\sum_{i=1}^{n} \tilde{\pi}\left(E_{i}\right)= & {\left[\nu\left(E_{1} \cup \ldots \cup E_{n}\right)-\nu\left(E_{2} \cup \ldots \cup E_{n}\right)\right] } \\
& +\ldots .+\left[\nu\left(E_{n-1} \cup E_{n}\right)-\nu\left(E_{n}\right)\right]+\widetilde{\pi}\left(E_{n}\right) \\
= & \nu(E)-\nu\left(E_{n}\right)+\widetilde{\pi}\left(E_{n}\right) \\
= & \nu(E)-\nu(F)+\widetilde{\pi}(F) .
\end{aligned}
$$

Monotonicity of $\nu$ implies that $\tilde{\pi}(E) \geq 0$ for all $E \in \widehat{\mathcal{E}}$.

Let us check now:

$$
\widetilde{\pi}(E \cup F)=\widetilde{\pi}(E)+\widetilde{\pi}(F)
$$

for all $E, F \in \widehat{\mathcal{E}}$ such that $E \cap F=\emptyset, E \cup F \in \widehat{\mathcal{E}}$.

If $E \cup F \in \widehat{\mathcal{E}}-\widetilde{\mathcal{E}}$ then this follows directly from Case 2 .

If $E \cup F \in \widetilde{\mathcal{E}}$ then, for $G \in \widehat{\mathcal{E}}$ such that $G \cap(E \cup F)=\emptyset$ and $G \cup(E \cup F) \in \widehat{\mathcal{E}}$ one obtains

$$
\begin{aligned}
\tilde{\pi}(E \cup F) & =\nu(E \cup F \cup G)-\nu(G) \\
& =[\nu(E \cup F \cup G)-\nu(F \cup G)]+[\nu(F \cup G)-\nu(G)] \\
& =\widetilde{\pi}(E)+\widetilde{\pi}(F) .
\end{aligned}
$$

(b) Next we extend $\tilde{\pi}$ on all of $\mathcal{E}$ to a non-negative simple additive measure.

Clearly, defining $\widetilde{\pi}(E)=0$ for all $E \subseteq Z$ is consistent with the restricted additivity of $\widetilde{\pi}$, i.e. $\tilde{\pi}(E \cup F)=\widetilde{\pi}(E)+\widetilde{\pi}(F)$ for all $E, F \in \mathcal{E}$ such that $E \cap F=\emptyset, E \cup F \in \widehat{\mathcal{E}}$. Consider a finite partition of $S,\left\{E_{i} \in \widehat{\mathcal{E}} \mid i=1, \ldots, n\right\}$, and define $\widetilde{\pi}(S):=\sum_{i=1}^{n} \widetilde{\pi}\left(E_{i}\right)$. To check that $\tilde{\pi}(S)$ is well-defined, let $\left\{F_{j} \in \widehat{\mathcal{E}} \mid j=1, \ldots, m\right\}$ be another finite partition of $S$, then one obtains

$$
\begin{array}{r}
\tilde{\pi}(S):=\sum_{i=1}^{n} \tilde{\pi}\left(E_{i}\right)=\sum_{i=1}^{n}\left[\sum_{j=1}^{m} \tilde{\pi}\left(E_{i} \cap F_{j}\right)\right] \\
=\sum_{j=1}^{m}\left[\sum_{i=1}^{n} \tilde{\pi}\left(E_{i} \cap F_{j}\right)\right]=\sum_{j=1}^{m} \tilde{\pi}\left(F_{j}\right) .
\end{array}
$$

Let $\alpha:=\widetilde{\pi}(S)$. Clearly, $\alpha \geq 0$. 
It remains to prove that $\widetilde{\pi}(E \cup F)=\widetilde{\pi}(E)+\widetilde{\pi}(F)$ for all $E, F \in \widehat{\mathcal{E}}$ such that $E \cap F=\emptyset$, $E \cup F \in \widehat{\mathcal{E}}$. Since $\widehat{\mathcal{E}}$ contains at least three elements, one can assume, without loss of generality, that $E=E_{1} \cup E_{2}$ with $E_{1}, E_{2} \in \widehat{\mathcal{E}}, E_{1} \cap E_{2}=\emptyset$. Hence, $\widetilde{\pi}(E)=\widetilde{\pi}\left(E_{1}\right)+$ $\widetilde{\pi}\left(E_{2}\right)$ and, from the definition of $\widetilde{\pi}(S)$, the desired result follows.

(c)We prove that there exists $\lambda \in \mathbb{R}_{+}$such that $\nu(E)=\lambda+\widetilde{\pi}(E)$ for all $E \in \widehat{\mathcal{E}}$.

If $E \in \widehat{\mathcal{E}}-\widetilde{\mathcal{E}}$ then, from Case 2, there exists $A \in \widehat{\mathcal{E}}$ such that

$$
\nu(E)=\widetilde{\pi}(E)+\nu(A)-\widetilde{\pi}(A) .
$$

If $E \in \widetilde{\mathcal{E}}$ then there exists $F \in \widehat{\mathcal{E}}$ and $B \in \mathcal{E}$ such that $E \cup F \cup B=S$. Hence, one has

$$
\tilde{\pi}(E \cup F)=\nu(E \cup F)+\nu(B)-\tilde{\pi}(B)
$$

and, therefore,

$$
\widetilde{\pi}(E)+\widetilde{\pi}(F)=\nu(E \cup F)-\nu(E)+\nu(E)+\widetilde{\pi}(B)-\nu(B),
$$

which gives

$$
\nu(E)=\widetilde{\pi}(E)+\nu(B)-\widetilde{\pi}(B) .
$$

Hence,

$$
\nu(A)-\widetilde{\pi}(A)=\nu(B)-\tilde{\pi}(B)
$$

for all $A, B \in \widehat{\mathcal{E}}$.

It remains to check that the common value $\lambda:=\nu(A)-\widetilde{\pi}(A)$ is non-negative. Let $E, F \in \widehat{\mathcal{E}}$ satisfy Property (b) and consider $A \subset E$ and $B \subset F$. Applying Property (a) twice gives

$$
\begin{aligned}
0 & \leq \nu(E)+\nu(F)-\nu(E \cup F)=\nu(A)+\nu(B)-\nu(A \cup B) \\
& =\nu(A)-\widetilde{\pi}(A)=\lambda
\end{aligned}
$$

(d)We prove that $\alpha+\lambda \leq 1$.

By Property (c), there is $E, F \in \widehat{\mathcal{E}}$ such that

$$
0 \leq \bar{\nu}(E)+\bar{\nu}(F)-\bar{\nu}(E \cup F)
$$




$$
\begin{aligned}
& =1-\nu(S-E)-\nu(S-F)+\nu(S-(E \cup F)) \\
& =1-(\lambda+\widetilde{\pi}(S-E))-(\lambda+\widetilde{\pi}(S-F))+(\lambda+\widetilde{\pi}(S-(E \cup F))) \\
& =1-\lambda-\alpha .
\end{aligned}
$$

(e) Setting $\gamma:=1-\lambda-\alpha$, we finally obtain: $\nu(E)=\gamma \cdot \mu^{0}(E)+\lambda \cdot \mu^{1}(E)+(1-$ $\gamma-\lambda) \cdot \pi(E)$ for all $E \in \mathcal{E}$ where, for $1-\gamma-\lambda>0$, the probability measure $\pi(E)$ is defined by $\pi(E):=\frac{1}{1-\gamma-\lambda} \cdot \widetilde{\pi}(E)$.

\section{A.2 Proof of Lemma 4.1}

Proof. From Equation $(*)$, we get

$$
\begin{aligned}
q^{*}= & \frac{\gamma \cdot u^{\prime}(\underline{r} \cdot A) \cdot \underline{r}+(1-\gamma) \cdot \sum_{s \in S} \pi_{s} \cdot u^{\prime}\left(r_{s} \cdot A\right) \cdot r_{s}}{r \cdot\left[\gamma \cdot u^{\prime}(\underline{r} \cdot A)+(1-\gamma) \cdot \sum_{s \in S} \pi_{s} \cdot u^{\prime}\left(r_{s} \cdot A\right)\right]} \\
= & \frac{\gamma \cdot u^{\prime}(\underline{r} \cdot A) \cdot \underline{r}+(1-\gamma) \cdot\left[r \cdot q_{0}^{*} \cdot \sum_{s \in S} \pi_{s} \cdot u^{\prime}\left(r_{s} \cdot A\right)\right]}{r \cdot\left[\gamma \cdot u^{\prime}(\underline{r} \cdot A)+(1-\gamma) \cdot \sum_{s \in S} \pi_{s} \cdot u^{\prime}\left(r_{s} \cdot A\right)\right]} \\
< & \frac{\gamma \cdot u^{\prime}(\underline{r} \cdot A) \cdot r \cdot q_{0}^{*}+(1-\gamma) \cdot\left[r \cdot q_{0}^{*} \cdot \sum_{s \in S} \pi_{s} \cdot u^{\prime}\left(r_{s} \cdot A\right)\right]}{r \cdot\left[\gamma \cdot u^{\prime}(\underline{r} \cdot A)+(1-\gamma) \cdot \sum_{s \in S} \pi_{s} \cdot u^{\prime}\left(r_{s} \cdot A\right)\right]} \\
= & q_{0}^{*} .
\end{aligned}
$$

The inequality follows because

$$
r \cdot q_{0}^{*}=\frac{\sum_{s \in S} \pi_{s} \cdot u^{\prime}\left(r_{s} \cdot A\right) \cdot r_{s}}{\sum_{s \in S} \pi_{s} \cdot u^{\prime}\left(r_{s} \cdot A\right)}<\underline{r} .
$$

\section{A.3 Proof of Theorem 5.1}

We begin with an observation and a couple of preliminary results. The observation is that any act $f \in \mathcal{F}$ may be expressed as $\left[x_{1}\right.$ on $E_{1} ; \ldots ; x_{n}$ on $\left.E_{n}\right]$, where $\left\{E_{1}, \ldots, E_{n}\right\}$ is the coarsest finite $\succsim$-ordered partition of $S$ with respect to which $f$ measurable. By that we mean for any pair of states $s, t \in S$, if both $s$ and $t$ are in some $E \in\left\{E_{1}, \ldots, E_{n}\right\}$ 
then $f(s)=f(t)$, otherwise $f(s) \neq f(t)$. Furthermore for any $s \in E_{i}$ and $t \in$ $E_{j}, i<j$ implies $f(s) \succsim f(t)$. Throughout this proof, if an act is expressed in the form $\left[x_{1}\right.$ on $E_{1} ; \ldots ; x_{n}$ on $\left.E_{n}\right]$ then it should be taken as given that $x_{i} \succsim x_{i+1}$, for $i=1, \ldots, i-1$. We also note that Axiom 5 (Extreme Events Sensitivity) and Axiom 1 (Ordering) imply that if the preference relation expresses indifference between two comonotonic acts then indifference is preserved when those two acts are each mixed with a third act that is pairwise co-monotonic with both.

Lemma: (Comonotonic Independence of Indifference) Axiom 5 implies that $\succsim$ satisfies the following independence property for pairwise comonotonic acts. For any $\alpha \in[0,1]$ and any three acts $f, g, h \in \mathcal{F}$, that are pairwise comonotonic, if $f \sim g$ then $\alpha f \oplus$ $(1-\alpha) h \sim \alpha g \oplus(1-\alpha) h$.

Proof: From the pairwise co-monotonicity of $h$ with both $f$ and $g$, it follows that $h \in$ $\mathcal{E}(g) \cap \overline{\mathcal{F}}(g)$ and $h \in \mathcal{F}(f) \cap \overline{\mathcal{F}}(f)$. Hence Axiom 5 implies that $\alpha f \oplus(1-\alpha) h \succsim$ $\alpha g \oplus(1-\alpha) h$ and $\alpha g \oplus(1-\alpha) h \succsim \alpha f \oplus(1-\alpha) h$, as required.

Finally we report GMMS's result (2002, Proposition 6) that the triple $(X, \sim, \oplus)$ constitutes a mixture set. That is, for all $x, y \in X$ and all $\alpha, \beta$ in $[0,1],(\mathrm{M} 0) \alpha x \oplus(1-\alpha) y \subset X$, (M1) $x \in(1 x \oplus 0 y)$, (M2) $\alpha x \oplus(1-\alpha) y=(1-\alpha) y \oplus \alpha x$ (commutative law), and (M3) $\beta(\alpha x \oplus(1-\alpha) y) \oplus(1-\beta) y=\alpha \beta x \oplus(1-\alpha \beta) y$ (distributive law). Applying this result state by state, to Definition 5.2 (the definition of a subjective mixture of $f$ and $g$ with weight $\alpha$ in $[0,1])$ it readily follows that the triple $(\mathcal{F}, \sim, \oplus)$ is also a mixture set and hence exhibits the analogous properties.

\section{Proof of Theorem 5.1-}

\section{Sufficiency}

We first show that $\succsim$ has a CEU representation, Part (i), and then that the capacity is neo-additive, Part (ii).

Part $(i): \succsim$ admits a CEU-representation. Let $u($.$) and \nu($.$) be the continuous utility$ index and capacity of the canonical biseperable representation that from Proposition (5.1) 
we know $\succsim$ admits. Recall that $u($.$) represents \succsim$ restricted to the constant acts, and that $V\left(\left[x_{1}\right.\right.$ on $E ; x_{2}$ on $\left.\left.S-E\right]\right)=\nu(E) u\left(x_{1}\right)+(1-\nu(E)) u\left(x_{2}\right)$ represents $\succsim$ restricted to the set of acts that are measurable with respect to a two-element partition of $S$.

Fix, $f=\left[x_{1}\right.$ on $E_{1} ; \ldots ; x_{n}$ on $\left.E_{n}\right]$. For each $i=1, \ldots, n$, it follows from the definition of $\oplus$ and the connectedness of $X$, that there exists a unique $\lambda_{i} \in[0,1]$ for which $x_{i} \in$ $\lambda_{i} M \oplus\left(1-\lambda_{i}\right) 0$ and a unique $\nu_{i}$ for which

$$
\left[M \text { on } E_{1} \cup \ldots \cup E_{i} ; 0 \text { on } E_{i+1} \cup \ldots \cup E_{n}\right] \sim \nu_{i} M \oplus\left(1-\nu_{i}\right) 0 \text {. }
$$

Equation (3) implies that $1 \geq \lambda_{1} \geq \ldots \geq \lambda_{n} \geq 0$ and $0 \leq \nu_{1} \leq \ldots \leq \nu_{n-1} \leq 1$.

Hence we have, by construction and the mixture set properties of $(\mathcal{F}, \sim, \oplus)$ that

$$
\begin{aligned}
& f=\left[\begin{array}{cc}
x_{1} & \text { on } E_{1} \\
\vdots & \vdots \\
x_{n} & \text { on } E_{n}
\end{array}\right]=\left[\begin{array}{cc}
\lambda_{1} M \oplus\left(1-\lambda_{1}\right) 0 & \text { on } E_{1} \\
\vdots & \vdots \\
\lambda_{n} M \oplus\left(1-\lambda_{n}\right) 0 & \text { on } E_{n}
\end{array}\right] \\
& =\left(1-\lambda_{1}\right)\left[\begin{array}{cc}
0 & \text { on } E_{1} \\
0 & \text { on } E_{2} \\
0 & \text { on } E_{3} \\
\vdots & \vdots \\
0 & \text { on } E_{n-1} \\
0 & \text { on } E_{n}
\end{array}\right] \oplus\left(\lambda_{1}-\lambda_{2}\right)\left[\begin{array}{cc}
M & \text { on } E_{1} \\
0 & \text { on } E_{2} \\
0 & \text { on } E_{3} \\
\vdots & \vdots \\
0 & \text { on } E_{n-1} \\
0 & \text { on } E_{n}
\end{array}\right] \oplus\left(\lambda_{2}-\lambda_{3}\right)\left[\begin{array}{cc}
M & \text { on } E_{1} \\
M & \text { on } E_{2} \\
0 & \text { on } E_{3} \\
\vdots & \vdots \\
0 & \text { on } E_{n-1} \\
0 & \text { on } E_{n}
\end{array}\right] \\
& \oplus \cdots \oplus\left(\lambda_{n-1}-\lambda_{n}\right)\left[\begin{array}{cc}
M & \text { on } E_{1} \\
M & \text { on } E_{2} \\
M & \text { on } E_{3} \\
\vdots & \vdots \\
M & \text { on } E_{n-1} \\
0 & \text { on } E_{n}
\end{array}\right] \oplus \lambda_{n}\left[\begin{array}{cc}
M & \text { on } E_{1} \\
M & \text { on } E_{2} \\
M & \text { on } E_{3} \\
\vdots & \vdots \\
M & \text { on } E_{n-1} \\
M & \text { on } E_{n}
\end{array}\right]
\end{aligned}
$$

By applying the comonotonic independence of indifference property of Lemma $6.3 n-1$

times and utilizing the distributive law of $(\mathcal{F}, \sim, \oplus)$, we obtain

$$
\begin{aligned}
f \sim & \left(1-\lambda_{1}\right) 0 \oplus\left(\lambda_{1}-\lambda_{2}\right)\left[\nu_{1} M \oplus\left(1-\nu_{1}\right) 0\right] \oplus\left(\lambda_{2}-\lambda_{3}\right)\left[\nu_{2} M \oplus\left(1-\nu_{2}\right) 0\right] \\
& \oplus \ldots \oplus\left(\lambda_{n-1}-\lambda_{n}\right)\left[\nu_{n-1} M \oplus\left(1-\nu_{n-1}\right) 0\right] \oplus \lambda_{n} M \\
= & {\left[\sum_{i=1}^{n-1}\left(\lambda_{i}-\lambda_{i+1}\right) \nu_{i}+\lambda_{n}\right] M \oplus\left[1-\lambda_{n}-\sum_{i=1}^{n-1}\left(\lambda_{i}-\lambda_{i+1}\right) \nu_{i}\right] \delta_{0} . }
\end{aligned}
$$


Hence it follows from equation (3) that for any pair of acts

$$
f=\left[\begin{array}{cc}
x_{1} & \text { on } E_{1} \\
\vdots & \vdots \\
x_{n} & \text { on } E_{n}
\end{array}\right] \text { and } f^{\prime}=\left[\begin{array}{cc}
x_{1}^{\prime} & \text { on } E_{1}^{\prime} \\
\vdots & \vdots \\
x_{n^{\prime}}^{\prime} & \text { on } E_{n^{\prime}}^{\prime}
\end{array}\right]
$$

applying the above methods we have

$$
\begin{aligned}
f & \succsim f^{\prime} \text { if and only if } \\
{\left[\sum_{i=1}^{n-1}\left(\lambda_{i}-\lambda_{i+1}\right) \nu_{i}+\lambda_{n}\right] } & \geq\left[\sum_{j=1}^{n^{\prime}-1}\left(\lambda_{j}^{\prime}-\lambda_{j+1}^{\prime}\right) \nu_{i}^{\prime}+\lambda_{n}^{\prime}\right]
\end{aligned}
$$

By construction, $u(0)=0, u(M)=1, u\left(x_{i}\right)=\lambda_{i}, \nu(\emptyset):=0, \nu(S):=1$ and $\nu\left(\cup_{j=1}^{i} E_{i}\right)=\nu_{i}$. Thus we have established that $\succsim$ can be represented by the Choquet expected utility functional

$$
\mathrm{CEU}\left(\left[\begin{array}{cc}
x_{1} & \text { on } E_{1} \\
\vdots & \vdots \\
x_{n} & \text { on } E_{n}
\end{array}\right]\right)=\sum_{i=1}^{n-1}\left(u\left(x_{i}\right)-u\left(x_{i+1}\right)\right) \nu\left(\cup_{j=1}^{i} E_{j}\right)+u\left(x_{n}\right)
$$

as required.

Part (ii): $\nu$ satisfies conditions (ii) of Proposition 3.1.

(a) We prove that for any three events $(E, F, G) \in \mathcal{E}^{*} \times \mathcal{E}^{*} \times \mathcal{E}^{*}$ such that $E \cap F=\emptyset=$ $E \cap G, E \cup F \notin \mathcal{U}, E \cup G \notin \mathcal{U}$.

$$
\nu(E \cup F)-\nu(F)=\nu(E \cup G)-\nu(G),
$$

Since there are at least four pairwise disjoint events in $\mathcal{E}^{*}$, we can assume that there are $E, F, G \in \mathcal{E}^{*} \times \mathcal{E}^{*} \times \mathcal{E}^{*}$ such that $E \cap F=E \cap G=F \cap G=\emptyset$ and $E \cup F \cup G \notin \mathcal{U}$. The following lemma contains the key argument.

Lemma: If there are $\mathcal{E}^{*} \times \mathcal{E}^{*} \times \mathcal{E}^{*}$ such that $E \cap F=E \cap G=F \cap G=\emptyset$ and $E \cup F \cup G \notin \mathcal{U}$. Then

$$
\nu(E \cup F \cup G)-\nu(F \cup G)=\nu(E \cup F)-\nu(F) .
$$

Proof: Assume, without loss of generality, $\nu(E \cup F) \leq \nu(F \cup G)$ and let $\beta \in[0,1]$ be such that $\nu(E \cup F)=\beta \cdot \nu(F \cup G)$. Consider

$$
f:=\left[\begin{array}{lll}
M & \text { on } & E \cup F \\
0 & \text { on } & S-(E \cup F)
\end{array}\right] \text {, }
$$




$$
\begin{aligned}
g: & =\left[\begin{array}{lll}
\beta \cdot M \oplus(1-\beta) \cdot 0 & \text { on } & F \cup G \\
0 & \text { on } & S-(F \cup G)
\end{array}\right], \\
h: & =\left[\begin{array}{lll}
M & \text { on } & F \cup G \\
0 & \text { on } & S-(F \cup G)
\end{array}\right] .
\end{aligned}
$$

Clearly, $f \sim g, h \in \underline{\mathcal{F}}(f) \cap \overline{\mathcal{F}}(f)$ and $h \in \underline{\mathcal{F}}(g) \cap \overline{\mathcal{F}}(g)$. By Axiom 5, Extreme Event Sensitivity, we have

$$
\frac{1}{2} \cdot g \oplus \frac{1}{2} \cdot h \sim \frac{1}{2} \cdot f \oplus \frac{1}{2} \cdot h
$$

Hence,

$$
\begin{aligned}
\frac{1}{2} \cdot[\nu(E \cup F \cup G)+\nu(F)] & =\frac{1}{2} \cdot(1+\beta) \cdot \nu(F \cup G) \\
& =\frac{1}{2} \cdot(\nu(F \cup G)+\nu(E \cup F)) .
\end{aligned}
$$

Thus, we conclude $\nu(E \cup F \cup G)-\nu(F \cup G)=\nu(E \cup F)-\nu(F)$.

Let us now show that $(E, F, G) \in \mathcal{E}^{*} \times \mathcal{E}^{*} \times \mathcal{E}^{*}$ such that $E \cap F=\emptyset=E \cap G$, $E \cup F \notin \mathcal{U}, E \cup G \notin \mathcal{U}$ implies

$$
\nu(E \cup F)-\nu(F)=\nu(E \cup G)-\nu(G) .
$$

Several cases have to be considered when $F \neq G$.

Case 1.1: $F \subset G$. Using Equation (*), we get

$$
\begin{aligned}
\nu(E \cup G)-\nu(G) & =\nu(E \cup F \cup(G-F))-\nu(F \cup(G-F)) \\
& =\nu(E \cup F)-\nu(F) .
\end{aligned}
$$

Case 1.2: $G \subset F$. Similar to Case 1.1.

Case 2.1: $F-G \neq \emptyset \neq G-F$ and $F \cap G \neq \emptyset$ Using Equation (*), we get

$$
\begin{aligned}
\nu(E \cup F)-\nu(F) & =\nu(E \cup(F \cap G) \cup(F-G))-\nu((F \cap G) \cup(F-G)) \\
& =\nu(E \cup(F \cap G))-\nu(F \cap G) \\
& =\nu(E \cup(F \cap G) \cup(G-F))-\nu((F \cap G) \cup(G-F)) \\
& =\nu(E \cup G)-\nu(G) .
\end{aligned}
$$

Case 2.2: $F-G \neq \emptyset \neq G-F$ and $F \cap G=\emptyset$ If $E \cup F \cup G \notin \mathcal{U}$, then the result follows immediately from Equation (*). 
Suppose $E \cup F \cup G \in \mathcal{U}$. Since $\mathcal{E}^{*}$ contains at least four elements we may assume that one of the events $E, F$ or $G$ can be partitioned into two events. Without loss of generality, suppose $E$ can be partitioned into $E_{1}$ and $E_{2}$. Then Equation (*) implies

$$
\begin{aligned}
\nu(E \cup F)-\nu(F) & =\nu\left(E_{1} \cup E_{2} \cup F\right)-\nu(F) \\
& =\left[\nu\left(E_{1} \cup E_{2} \cup F\right)-\nu\left(E_{1} \cup F\right)\right]+\left[\nu\left(E_{1} \cup F\right)-\nu(F)\right] \\
& =\left[\nu\left(E_{2} \cup F\right)-\nu(F)\right]+\left[\nu\left(E_{1} \cup F\right)-\nu(F)\right] \\
& =\left[\nu\left(E_{2} \cup G\right)-\nu(G)\right]+\left[\nu\left(E_{1} \cup G\right)-\nu(G)\right] \\
& =\left[\nu\left(E_{1} \cup E_{2} \cup G\right)-\nu\left(E_{1} \cup G\right)\right]+\left[\nu\left(E_{1} \cup G\right)-\nu(G)\right] \\
& =\nu(E \cup G)-\nu(G) .
\end{aligned}
$$

(b) We prove that for some $(E, F) \in \mathcal{E}^{*} \times \mathcal{E}^{*}$ such that $E \cap F=\emptyset$ and $E \cup F \notin \mathcal{U}$,

$$
\nu(E \cup F) \leq \nu(E)+\nu(F) .
$$

Consider $(E, F) \in \mathcal{E}^{*} \times \mathcal{E}^{*}$ such that $E \cap F=\emptyset$ and $E \cup F \notin \mathcal{U}$. Assume, without loss of generality, that $\nu(E)=\beta \cdot \nu(F)$ for some $\beta \in[0,1]$. Let

$$
\begin{aligned}
f: & =\left[\begin{array}{lll}
M & \text { on } & E \\
0 & \text { on } & S-E
\end{array}\right], \\
g:= & {\left[\begin{array}{lll}
\beta \cdot M \oplus(1-\beta) \cdot 0 & \text { on } & F \\
0 & \text { on } & S-F
\end{array}\right], } \\
h:= & \left.\begin{array}{lll}
M & \text { on } & F \\
0 & \text { on } & S-F
\end{array}\right] .
\end{aligned}
$$

Clearly, $f \sim g, h \in \mathcal{F}(f)$ and $h \in \mathcal{E}(g) \cap \overline{\mathcal{F}}(g)$. By Axiom 5(1), Extreme Event Sensitivity, we have

$$
\frac{1}{2} \cdot g+\frac{1}{2} \cdot h \succeq \frac{1}{2} \cdot f+\frac{1}{2} \cdot h
$$

Hence,

$$
\begin{aligned}
\frac{1}{2} \cdot \nu(E \cup F) & \leq \frac{1}{2} \cdot(1+\beta) \cdot \nu(F) \\
& =\frac{1}{2} \cdot(\nu(F)+\nu(E)) .
\end{aligned}
$$

Thus, we conclude $\nu(E \cup F) \leq \nu(E)+\nu(F)$. 
(c) We prove that for some $(E, F) \in \mathcal{E}^{*} \times \mathcal{E}^{*}$ such that $E \cap F=\emptyset$ and $E \cup F \notin \mathcal{U}$,

$$
\bar{\nu}(E \cup F) \leq \bar{\nu}(E)+\bar{\nu}(F)
$$

Consider $(E, F) \in \mathcal{E}^{*} \times \mathcal{E}^{*}$ such that $E \cap F=\emptyset$ and $E \cup F \notin \mathcal{U}$. Assume, without loss of generality, that $\nu(S-E)=\beta \cdot \nu(S-F)$ for some $\beta \in[0,1]$. Let

$$
\begin{aligned}
f: & =\left[\begin{array}{lll}
M & \text { on } & S-E \\
0 & \text { on } & E
\end{array}\right], \\
g: & =\left[\begin{array}{lll}
\beta \cdot M \oplus(1-\beta) \cdot 0 & \text { on } & S-F \\
0 & \text { on } & F
\end{array}\right], \\
h: & =\left[\begin{array}{lll}
M & \text { on } & S-F \\
0 & \text { on } & F
\end{array}\right] .
\end{aligned}
$$

Clearly, $f \sim g, h \in \overline{\mathcal{F}}(f)$ and $h \in \mathcal{F}(g) \cap \overline{\mathcal{F}}(g)$. By Axiom 5(2), Extreme Event Sensitivity, we have

$$
\frac{1}{2} \cdot f+\frac{1}{2} \cdot h \succeq \frac{1}{2} \cdot g+\frac{1}{2} \cdot h
$$

Hence,

$$
\begin{aligned}
\frac{1}{2}+\frac{1}{2} \cdot \nu((S-E) \cup(S-F)) & \geq \frac{1}{2} \cdot(1+\beta) \cdot \nu(S-F) \\
& =\frac{1}{2} \cdot(\nu(S-F)+\nu(S-E))
\end{aligned}
$$

or

$$
\frac{1}{2} \cdot[(1-\nu(S-F))+(1-\nu(S-E))] \geq \frac{1}{2} \cdot[1-\nu((S-E) \cup(S-F))] .
$$

Thus, we conclude $\bar{\nu}(E \cup F) \leq \bar{\nu}(E)+\bar{\nu}(F)$.

\section{Necessity.}

The necessity of the representation follows straightforwardly from the definition of the neo-additive representation and so the proof is omitted. 


\section{References}

Allais, M. (1953). "Le comportement de l' homme rationnel de vant le risque: Critique des postulats et axioms de l'école Americaine". Econometrica 21, 503-546.

Abdellaoui, M. (2000). "Parameter-Free Elicitation of Utility and Probability Weighting Functions”. Management Science 46, 1497-1512.

Anscombe, F.J. \& Aumann, R.J. (1963). "A Definition of Subjective Probability”. Annals of Mathematical Statistics 34, 199-205.

BeLL, D.E. (1985). "Disappointment in Decision Making under Uncertainty". Operations Research 33, 1-27.

Bleichrodt, H., \& Pinto, J.L. (2000). "A parameter-Free Elicitation of the Probabaility Weighting Function in Medical Decision Analysis". Management Science 46, $1485-1496$.

Chew, Soo Hong And Edi Karni (1994). "Choquet expected utility with a finite state space: commutativity and act-independence", Journal of Economic Theory, 62, 469479.

Cohen, M. (1992). "Security Level, Potential Level, Expected Utility: A ThreeCriteria Decision Model under Risk". Theory and Decision 33, 101-134.

De Finetti, B. (1937). "La prevision: ses lois logiques, ses sources subjectives". Annales de l'Institut Henri Poincaré 7, 1-68. (English translation in KYBURG, H.E. Jr. \& Smokler, H.E., Studies in Subjective Probability. New York: John Wiley and Sons, 1964).

Denneberg, D. (2000). "Non-additive Measure and Integral, Basic Concepts and Their Role for Applications". In Grabisch, M., Murofushi, T. \& Sugeno, M. (EDS. ), Fuzzy Measures and Integrals. Theory and Applications. Heidelberg, New York: Physica Verlag.

Eichberger, J. \& Kelsey, D. (2000). "Non-Additive Beliefs and Strategic Equilibria”. Games and Economic Behaviour 30, 182-215.

Eichberger, J. \& Kelsey, D. (1999). "E-Capacities and the Ellsberg Paradox". Theory and Decision 46, 107-140.

EllsBerg, D. (1961). "Risk, Ambiguity and the Savage Axioms". Quarterly Journal of Economics 75, 643-669.

FishbuRn, P.C. (1970). Utility Theory for Decision Making. Reprinted Edition 1979. New York: Robert Krieger Publishing Company.

Friedman, M. \& Savage, L.J. (1948). "The Utility Analysis of Choices Involving Risk". Journal of Political Economy 56, 279-304. 
Ghirardato, P. \& Marinacci, M. (2001). "Risk, Ambiguity, and the Separation of Utility and Beliefs", Mathematics of Operations Research 26, 864-890.

Ghirardato, P., Maccheroni, F., \& Marinacci, M. (2002). "Ambiguity from the Differential Viewpoint". Calfornia Institute of Technology Social Science Working Paper 1130.

Ghirardato, P., Maccheroni, F., Marinacci, M. \& Siniscalchi, M. (2001). "A Subjective Spin on Roulette Wheels", Calfornia Institute of Technology Social Science Working Paper 1127R.

GilboA, I. (1988). “A Combination of Expected Utility and Maxmin Decision Criteria”. Journal of Mathematical Psychology 32, 405-420.

Gonzalez, R. \& Wu, G. (1999). "On the Shape of the Probability Weighting Function”. Cognitive Psychology 38, 129-166.

Gul, FARUK (1992). "Savage's Theorem with a Finite Number of States”, Journal of Economic Theory 57, 99-110.

Hirshleifer, J. \& Riley, J.C. (1992). The Analytics of Uncertainty and Information. Cambridge, UK: Cambridge University Press.

JAFFrAY, J.-Y. (1988). "Choice under Risk and the Security Factor: An Axiomatic Model". Theory and Decision 24, .

Keynes, J.M. (1921). A Treatise on Probability. London: Macmillan.

Kilka, M. \& Weber, M. (1998). "What Determines the Shape of the Probability Weighting Function under Uncertainty?” Management Science 47, 1712-1726.

Kopylov, I. (2002). “ $\alpha$-Maxmin Expected Utility”. Mimeograph.

Machina, M. \& Schmeidler, D. (1992). "A More Robust Definition of Subjective Probability”. Econometrica 60, 745-780.

Markowitz, H. (1952). "The Utility of Wealth". Journal of Political Economy 60, 151-158.

Mehra, R. \& Prescott, E. (1985). “The Equity Premium: A Puzzle”. Journal of Monetary Economics 15, 145-161.

Nakamura, Yutaka (1990). "Subjective Expected Utility with Non-additive Probabilities on Finite State Spaces", Journal of Economic Theory 51, 346-366.

RAMSEY, F.P. (1926). "Truth and Probability”. In: The Foundations of Mathematics and Other Logical Essays. New York: Harcourt,Brace and Co., 1931.

SARIN, R. \& WAKKeR, P. (1998). "Revealed Likelihood and Knightian Uncertainty". Journal of Risk and Uncertainty 16, 223-250.

Savage, L.J. (1954). The Foundations of Statistics. New York: Dover Publications 1972. 
SCHMEIDLER, D. (1989). "Subjective Probability and Expected utility without Additivity". Econometrica 57, 571-587.

ShIller, R.J. (2001). Irrational Exuberance. Paperback edition. New York: Broadway Books.

Smith, AdAm (1776). The Wealth of Nations. Edited by Andrew Skinner. Harmondsworth: Pelican Classics, 1976.

Thaler, R.H. (1994). The Winnwer's Curse. Paradoxes and anomalies of Economic Life. Princeton, N.J.: Princeton University Press.

Tversky, A., \& Wakker, P. (1995). "Risk Attitudes and Decision Weights". Econometrica 63, 1255-1280.

WAKkeR, P. (2001). "Testing and Characterizing Properties of Nonadditive Measures through Violations of the Sure-Thing Principle”. Econometrica 69, 1039-1059. 